\title{
Product-Line Assurance Cases from Contract-Based Design
}

Damir Nešić, Royal Institute of Technology, damirn@kth.se Mattias Nyberg, Royal Institute of Technology, matny@kth.se Barbara Gallina, Mälardalen University, barbara.gallina@mdh.se Submitted to: Elsevier Journal of Systems and Software.

\begin{abstract}
Assurance cases are used to argue in a structured, and evidence-supported way, that a property such as safety or security is satisfied by a system. In some domains however, instead of single systems, product lines with many system-variants are engineered, to satisfy the needs of different customers. In such context, singlesystem methods for assurance-case creation suffer from scalability issues because the underlying assumption is that the evidence and arguments can be created per system variant. This paper presents a novel method for product-line assurance-case creation where all the arguments and the evidence are created without analyzing each system variant. Consequently, the effort to create an assurance case scales with the complexity of system variants, instead with their number. The method is based on a contract-based design framework for cyber-physical systems, which is extended to define the conditions under which all system variants satisfy a particular property. These conditions are used to define an assurance-case pattern, which can be instantiated for arbitrary product lines. Moreover, the defined pat-
\end{abstract}


tern is modular to enable step-wise assurance-case creation. Finally, an exploratory case study is performed on a real product-line from the heavy-vehicle manufacturer SCANIA to evaluate the applicability of the presented method.

\section{Introduction}

An assurance case is "a documented body of evidence that provides a convincing and valid argument that a specified set of critical claims regarding a system's properties are adequately justified for a given application in a given environment" 69. Similar to a court case, an assurance case is a structured, evidencebased way to present the arguments that a particular system is acceptably safe, acceptably secure etc. Conceptually, an assurance case contains two types of arguments, product-based and process-based. The former should show that the system behaves in accordance with its specification, while the latter should show that the engineering personnel, processes, and tools cannot accidentally introduce flaws into the system. Currently, assurance cases are recommended or even required by various standards, e.g. ISO 26262 [48] or EN 50129 [26], including the recent, first-ever standard for the safety of autonomous systems, UL 4600 [8].

To support different operational environments, and satisfy the needs of different markets and corresponding legislative frameworks, companies typically develop families of similar systems, called Product Lines [57, 13], and not single systems. Examples of product lines are everywhere, from open-source software product-lines such as the Linux and eCos operating systems [44], to product lines in critical domains such as industrial automation [28, automotive [85], aerospace [29], railway 75] etc. Independently of the domain, product lines are typically designed as integrated platforms from which the members of the family, called variants, can be derived. Consequently, when creating assurance cases, companies are faced with the challenge of arguing that all variants are acceptably safe, secure etc., instead of the classical case with a single system.

Product lines come with two fundamental challenges that hinder the creation of corresponding assurance cases. Firstly, a significant number of product lines define thousands and even millions of possible system variants 62, 60, 85, 22, Consequently, exhaustive verification of all derivable system variants is typically not feasible because the cost of deriving and verifying each system variant is too high. For this reason, verification evidence cannot be produced for each system variant and creating an assurance case per system variant is not a feasible option. 
The second challenge relates to the analysis of product-line assets. Typically, engineering assets of a product line are created so that they apply to multiple system variants. For example, a piece of source code could belong to many variants, but each variant will execute only a part of the source code. The consequence is that the classical, single-system analysis methods can only be used if an asset is reduced to a version that belongs to a single variant. To avoid this overhead, different analysis methods are modified to become variabilityaware [77, i.e. they are designed to analyze an asset as-is by considering the built-in variability. Preferably, the same principles should exist for an assurance case that argues about multiple variants, i.e. there should exist a set of variability-aware conditions that define if an assurance case is complete, correct etc.

\subsection{Main Related Work}

This section reviews existing methods that tackle the problem of creating an assurance case for a product line, to highlight their limitations. Methods that deal with parts of this problem, e.g. how to represent the relevant artifacts of a product line, or how to create assurance-case arguments that can be reused in different contexts, are considered in Section 9.

There are two notable lines of research for systematic creation of assurance cases for product lines where both focus on per-variant assurance-case creation, with primarily product-based arguments about safety properties.

The first line of research [38, 41, 18] presents a generic, product-line safety meta-model that defines generic traceability links and generic assets, which can be common or variable. Examples of traceability links are associated with, or generated from, while safety analysis assets or assurance-case assets are examples of generic assets. The meta-model can then be used to structure concrete assets in a concrete domain to support traceable product-line engineering process. Furthermore, the Goal-Structuring Notation (GSN) [68] is used to express assurance-case arguments, which reflect the variability within the product-line assets. In other words, the method yields an integrated product-line assurancecase argument that argues about multiple system variants, in the same way as product-line assets belong to multiple system variants. Then, just as system variants are derived from the product line, per variant assurance-case can be derived from the integrated product-line assurance case.

While the work in [38, 41, 18] supports more systematic product-line safety analyses, and the creation of an integrated product-line assurance case, the two fundamental challenges of product line engineering are not addressed. Firstly, 
the method assumes that per-variant assurance cases will be derived, and analyzed for completeness and consistency, i.e. there are no variability-aware criteria to analyze the assurance case. It follows that the integrated assurance case is essentially just a superimposition of multiple per-variant assurance cases. Secondly, as a consequence of the first issue, the verification evidence must be created per system-variant which, as discussed previously, is typically not feasible.

The second line of research [45, 46, 47] focuses on the aerospace domain, and considers pure software assurance. The method relies on a model-to-model transformation from a UML class-model of a software system, to SPARK Ada programs [9]. The UML class-models contain parametrized annotations, which define the properties that different system variants must satisfy. During model-tomodel transformation, which corresponds to variant derivation, all parametrized annotations are resolved, and a particular system variant is derived. Then, offthe-shelf tools can be used to automatically analyze per-variant SPARK Ada programs in order to guarantee data-flow properties, functional correctness etc. In other words, the method allows producing evidence which supports productbased arguments that a software variant satisfies a certain property. As an extension, the work in [47] discusses the notion of trustworthiness, and defines several process-based arguments, which are required to claim that each variant derived from a product line is safe.

The notion of trustworthiness in [45, 46, 47], coupled with SPARK Ada definitions of a component satisfying a property, explicitly shows why the resulting arguments are complete and correct. However, although automatically, both the verification evidence and the analysis for completeness and correctness is still performed on a per variant basis. Furthermore, because properties such as safety or security are system-level properties, the relation between the claim that a software component will operate as intended, and the claim that the overall system containing the software will operate as intended, is undefined.

\subsection{Paper Contribution}

Given the challenges of product line engineering, and the limitations of previous method for assurance-case creation for product lines, the goal of the present paper is to define a novel and general method for the creation of assurance-case argumentation for all variants that can be derived from a product line, without actually performing the derivation.

To this end, the current paper presents a method based on a formal ContractBased Design (CBD) framework [82, 8, 11. Because existing CBD frameworks were developed for single system analysis, through a series of formal definitions, 
theorems, and deductive proofs, the first contribution of the paper is a set of variability-aware conditions over an extended CBD framework, which preserve the compositional nature of the original CBD framework, but allow modeling and analysis of product lines. If satisfied, these conditions allow inferring that all possible system variants satisfy the declared system property. Furthermore, the combination of the variability-aware conditions, and the compositional nature of the CBD framework, means that instead of verifying a high number of variants, it is sufficient to verify only the components that comprise the different variants.

The second contribution is the definition of two assurance-case patterns [54] in the GSN format, which given a CBD model of a product line, create the corresponding product-based, assurance-case argumentation. More precisely, the assurance-case patterns are based on the variability-aware conditions of the extended CBD framework. In this way, the argumentation obtained by instantiating the pattern argues directly about all possible variants of a system. Because for real systems, the assurance-case argumentation can be quite large, the presented patterns are modular. This supports both the separation of concerns, e.g. different engineers can create smaller parts of the assurance case, and also a stepwise creation of the assurance case in parallel with the engineering process.

To verify if the extended CBD framework can be used to model and analyze real product lines, the paper presents an exploratory case study [72] by applying the framework to a real, configurable system from the heavy vehicle manufacturer SCANIA. The case study reveals that the CBD framework can indeed be applied to a real system, but depending on the available artifacts and used engineering practices, some information must be inferred by jointly analyzing the conditions of the CBD framework and the available artifacts. To evaluate the assurance-case patterns, the analyzed SCANIA system is used to instantiate the pattern. Despite the need for further validation, initial results show that the proposed modular architecture aligns rather well with the analyzed engineering process, and with the process of the functional safety standard from the automotive domain, i.e. ISO 26262. Regarding the types of evidence required by the pattern, the majority was already present among the SCANIA artifacts. However, two types were not because producing them requires more advanced, yet mature, automated methods.

The present paper extends the work in 65 by considering a more expressive contract-based design-framework in order to support a greater array of engineering scenarios. Consequently, the product-line extension of the contract-based framework is also refined. Furthermore, and unlike [65], an exploratory case study is performed on a part of a real product-line from the heavy vehicle manufacturer SCANIA, to evaluate the applicability of the extended contract-based 
framework. Finally, the assurance-case pattern is refined in order to match the refined extension of the contract-based framework, and the pattern is redefined to yield a modular assurance case.

\subsection{Paper Structure}

Section 6.1 presents the underlying methodology of the present paper. Section 3 summarizes the basics of product lines and an existing contract-based framework. Section 4 presents minor modifications of the existing framework in order to allow explicit modeling of assumptions that can later be considered in the product line extension. Section 5 presents the product-line extension of the general contract-based framework, which allows modeling of configurable systems. Section 6 presents an exploratory case study that evaluates the applicability of the CBD framework to a configurable system from ScANIA. Section 7 recalls the basics of GSN format and then defines the modular assurance-case pattern. This is followed by a pattern-instantiation procedure and an example instantiation for the analyzed SCANIA system. Section 8 answers the two research question. Section 9 discusses the related work while Section 10 reviews the threats to validity for the presented method. Finally, Section 11 concludes the paper.

\section{Methodology}

This section discusses the methodological foundation of the present paper and its visualization is shown in Figure 5.1. As outlined in Section 1, the goal of the present paper is to define a general method for the creation of assurance-case argumentation for all variants that can be derived from a product line, without actually performing the derivation. Similarly to the methods that produce pervariant assurance-case argumentation, this entails creating a model of a product line and its artifacts, and a subsequent definition of a method that leverages the model to create assurance-case argumentation.

As Figure 5.1 shows, the present paper relies on a CBD framework [82, 8, 11] to model an arbitrary system, i.e. a configurable system. There are multiple reasons for this choice. The most important reason is the support for compositional reasoning [19] and compositional verification [7, i.e. the properties of a system can be inferred from the verified properties of its constituent components. Consequently, the properties of individual variants can be inferred from the properties of their constituent components, without actually deriving indi- 


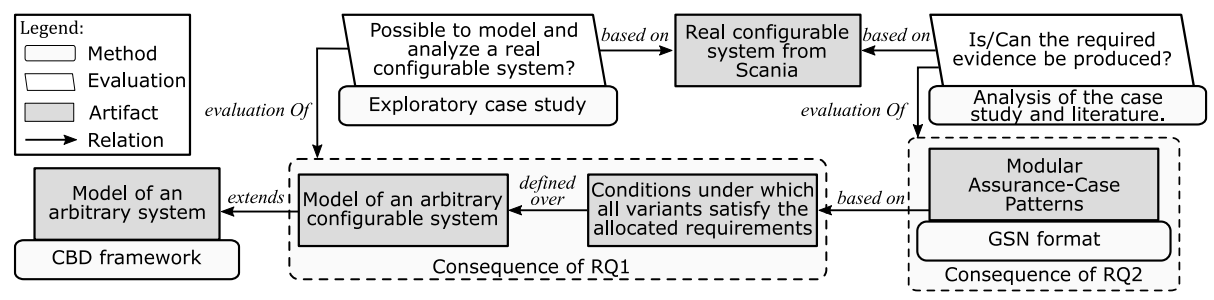

Figure 5.1: Underlying methodology and relations between the elements of the present paper.

vidual variants. Another important reason is that CBD frameworks formalize the typical engineering process as-is [8], i.e. they formalize in an abstract way the vertical design refinement during the development process, and horizontal composition of logical or physical components at a given abstraction level. However, CBD frameworks are not intended to be a concrete engineering framework, but rather an orthogonal representation of engineering artifacts, which enables advanced, and preferably automated analyses regardless of the used engineering practices. This is achieved by abstracting away the exact content of the artifacts and defining refinement and composition independently of the concrete syntax used to express the different artifacts. Furthermore, contract thinking is not uncommon in everyday engineering, nor new in general. First explicit contract-driven method for software development dates back to early '90s 61. Furthermore, ten years ago, ISO 26262 has introduced the concept of a safety element out of context, that is developed with respect to a contract and it guarantees certain functionality only when it is integrated with other systems that satisfy certain assumptions.

Despite the benefits, none of the existing CBD frameworks support modeling of configurable systems (see survey in [8]), i.e. product lines, thus it was necessary to extend an existing CBD framework and this has led to the first research question:

RQ1 Can a CBD framework be used to model an arbitrary product line, and support an analysis of whether all variants satisfy a particular property without performing the analysis per-variant?

As shown in Figure 5.1, a consequence of RQ1 is the first contribution of the paper, namely the extension of an existing CBD framework with support to model and analyze configurable systems. To evaluate if that this is indeed 
possible, an exploratory case study [72] was performed on a real, configurable systems from the heavy-vehicle manufacturer SCANIA.

Given the extended CBD framework, and to reach the overall goal of the paper, it was necessary to define a method that leverages the extended CBD framework to construct assurance-case argumentation. This has led to the formulation of the second research question, namely:

RQ2 How can a model of a product line in the CBD framework be used to construct an assurance case for an arbitrary product line?

As illustrated in Figure 5.1 the developed method was encoded as several modular assurance-case patterns [54] expressed in GSN format. The GSN format was chosen because of an abundance of available tool-support, and because it is frequently used in academic publications. However, the encoded argumentation is in no way dependent on the format in which it is expressed. Because the modular assurance-case patterns are based on the conditions of the extended CBD framework, and because the expected assurance-case evidence corresponds to the successful verification results of these conditions, the proposed patterns are evaluated from the perspective of whether these conditions are practically verifiable. The analysis was done both against the identified artifacts and observed practices in ScANIA, but also against existing tool-supported methods that can verify such conditions.

\section{Preliminaries}

This section summarizes the basic concepts of product lines and CBD frameworks from previous literature.

\subsection{Product Line Engineering}

Product line engineering [57, 3], facilitates the development of a family of systems that are jointly referred to as a product line. Instead of developing each system individually, product line engineering promotes the creation of an integrated platform that contains the artifacts of all variants, and then individual variants are derived from the integrated platform. To distinguish between variants on a more abstract level than the corresponding artifacts, product line engineering promotes the use of features. Features are abstractions of functional and non-functional characteristics of each system variant, that are understandable 
for the customers of the system. For example, automatic and manual transmission could be features within an automotive product-line. Features, and their dependencies, are captured through variability models.

A product line can be implemented in many ways [24, 56] but there is a wide agreement that independently of the selected technologies, the mechanisms underlying product line implementation is either annotative or compositional [53]. In annotation-based product lines, each artifact is annotated with the features to which it applies. By selecting a set of features, the corresponding artifacts are indirectly selected, and a system variant is derived. In compositional product lines, the implementation of each feature is a single 'unit', e.g. a particular software class, and when a set of features is selected, the corresponding units are composed into a system variant.

Compositional approaches are suitable for software product lines where a compiler guarantees the correct composition of source-code units. However, composition of other artifacts is not always defined, and rarely tool-supported, e.g. composition of requirements. Thus, product lines are typically implemented using the annotative mechanism, which is the one considered in the remainder of the paper.

\section{Annotation-Based Product Lines}

Let $\mathcal{F}$ be a set of features of a product line where each feature $f_{i} \in \mathcal{F}$ is a Boolean variable. Features can also be of other types, e.g. numerical or enumerations, but this is a trivial extension and is not considered further to avoid notational overhead.

Definition 1 (Variability Model). A variability model is a pair $\mathfrak{M}=(\mathcal{F}, \Theta)$ where $\mathcal{F}$ is a set of features and $\Theta$ is a set of Boolean formulas over the features in $\mathcal{F}$.

There are multiple types of variability models introduced in the literature [15], but the de-facto standard is a feature model [66]. The idea behind a feature model, as shown in Figure 5.2a is to visualize the features, and a subset of the Boolean formulas as a tree-structure that is intuitive to build, understand, and maintain. Within a feature model, features can be declared to be mandatory, optional etc., and Figure $5.2 \mathrm{~b}$ shows how the different feature-model constructs are encoded as Boolean formulas within $\Theta$. For example, features $f_{4}$ and $f_{5}$ could represent the automatic and manual transmission, that are mutually exclusive, while their parent feature $f_{2}$ could represent transmission feature that is mandatory. As Figure 5.2a shows, additional Boolean formulas outside the 
tree-structure are maintained in a textual format, e.g. $\theta_{i}$ and $\theta_{j}$ in Figure 5.2a, Variability models allow the selection of configurations.

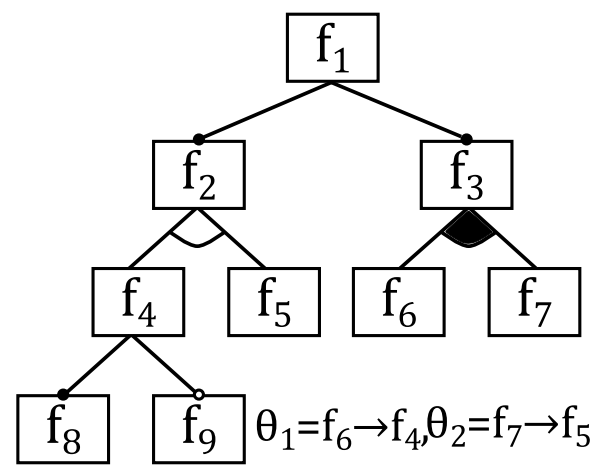

(a)

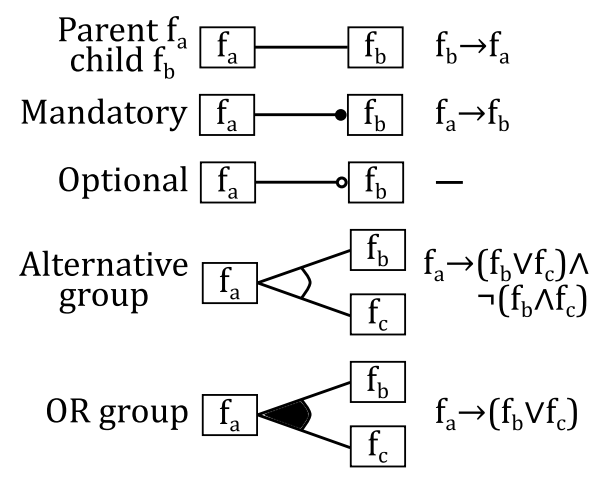

(b)

Figure 5.2: Example feature model (a), and the corresponding encoding as Boolean formulas (b).

Definition 2 (Configuration). Given a variability model $\mathfrak{M}$, a configuration $\gamma$ is a set of feature-value assignments $\gamma=\left\{f_{i}=\right.$ value $\left._{k}\right\}$ for each $f_{i} \in \mathcal{F}$. A configuration for which each formula in $\Theta$ evaluates to true is valid, and the set of valid configurations is denoted $\Gamma$.

The previously informal notion of selecting a feature corresponds to assigning the value true to a feature, while de-selecting corresponds to assigning the value false. If a value is assigned to each of the features from $\mathcal{F}$ then a configuration is selected. To highlight an invalid configuration, consider a $\gamma$ for the feature model in Figure 5.2 where $\left\{f_{2}=\right.$ true,$f_{4}=$ true, $f_{5}=$ true $\} \subseteq \gamma$. For such $\gamma$, the constraint for the alternative group $f_{2}, f_{4}, f_{5}$ would evaluate to false, thus $\gamma \notin \Gamma$. On an intuitive level, if $f_{4}$ and $f_{5}$ represent automatic and manual transmission, then the feature model in Figure 5.2 declares that a vehicle cannot simultaneously have both types of transmission. Hereinafter, we will consider only the set of valid configurations $\Gamma$. Variability models of real-world product lines often contain thousands of features, and consequently define billions of valid configurations 62 .

Given a variability model, the artifacts of the integrated platform are annotated with presence conditions [79]. 
Definition 3 (Presence condition). A presence condition, denoted $\varphi$, is a Boolean formula over the features from $\mathcal{F}$ from a variability model $\mathfrak{M}$, conforming to the grammar $\varphi::=f_{i}|\neg \varphi| \varphi \wedge \varphi \mid \varphi \vee \varphi$.

The purpose of a presence condition is to define a subset of $\Gamma$, to which an artifact applies. For example, if a presence condition is $f_{1} \wedge f_{4}$, then this presence condition evaluates to true for all configurations that contain value assignments $f_{1}=$ true and $f_{4}=$ true. Formally, let function eval be such that given a presence condition $\varphi$ and a configuration $\gamma$ it returns a value true or false. Note that we will consider only presence conditions that are consistent with the given variability model $\mathfrak{M}$, i.e. for each $\varphi$, there exists a $\gamma \operatorname{such}$ that $\operatorname{eval}(\varphi, \gamma)=$ true. For example, in the context of the feature model in Figure 5.2a this excludes the presence condition such as $f_{9} \wedge f_{10}$ because $f_{10}$ is not defined in the feature model.

The function eval models the so-called product configurator that allows a customer to select a configuration of the product in terms of features, and then evaluates the presence condition of all artifacts within the integrated platform. The artifacts whose presence conditions evaluate to true, are the ones that belong to the variant that corresponds to the selected configuration. To analyze the properties of each variant, e.g. safety or security, it is necessary to have a model of the artifacts of the integrated platform, e.g. logical and technical components, requirements etc.

\subsection{A Contract-Based Design Framework}

This section summarizes the used CBD concepts from 81, 8, with a slightly different notation that highlights the use of $\mathrm{CBD}$ for requirements engineering. The axiomatic concepts of the CBD framework are:

i) component $C$,

ii) specification $S$,

iii) component satisfies a specification, denoted as $C \triangleright S$

iv) specification refines a specification, denoted $S_{1} \sqsubseteq S_{2}$

v) commutative and associative n-ary component composition that results in new components, denoted as $C=C_{1} \otimes \cdots \otimes C_{n}$ or shortly $C=\bigotimes_{i=1}^{n} C_{i}$. 
The purpose of the above concepts is to represent central artifacts and activities during systems engineering. Components represent physical or logical components of a system, while the composition operation represents the common engineering activity of integrating components into systems. A specification defines the intended behavior of a component, and the satisfies relation represents the observation that a component behaves as defined by a specification. Specification refinement means that if a component $C$ satisfies $S_{1}$, and if $S_{1}$ refines $S_{2}$, then the component $C$ also satisfies $S_{2}$. Depending on the interpretation of specifications, refinement can correspond to logical entailment between logical formulas, to the subset relation between the sets of possible behaviors, etc. However, being axiomatic, no particular formalism is assumed for the above concepts. For example, a specification can be a temporal-logic formula, a differential equation, or plain natural-language text.

We refer to components that are not composed of other components as atomic components, and to components composed of other components as composite components. We also say that a composite component has subcomponents. To capture the composition of components into larger components, i.e. a system, we introduce the concepts of an architecture where the terminology related to trees, and graphs in general, is in accordance with [70].

Definition 4 (Architecture). An architecture $\mathscr{A}$ is a finite, non-empty set of components organized into a rooted tree where each leaf node is an atomic component, and each non-leaf node $C$ with children nodes $C_{1}, \ldots, C_{n}$ is a composite component, such that $C=\bigotimes_{i=1}^{n} C_{i}$.

Note that Def. 4 does not place any constraints on the types of components that can be composed into an architecture, e.g., SW and HW components can be composed into the same architecture. As stated above, specifications define the intended behavior of components. As is often the case, several specifications define the behavior of a component, and to capture this scenario we define specification conjunction.

Definition 5 (Specification conjunction). The conjunction of specifications $S_{1}$ and $S_{2}$, denoted $S_{1} \sqcap S_{2}$, is a specification such that $C \triangleright\left(S_{1} \sqcap S_{2}\right) \Leftrightarrow C \triangleright S_{1} \wedge$ $C \triangleright S_{2}$.

To be able to split the responsibilities on a component and on other components in its environment, the concept of a contract is introduced.

Definition 6 (Contract). A contract $K$ is an ordered pair, denoted $(\mathcal{A}, G)$, where $\mathcal{A}$ is a possibly empty, finite set of specifications called assumptions, i.e. $\mathcal{A}=\left\{A_{i}\right\}_{i}$, and $G$ is a specification called a guarantee. 
The contracts defined in Definition 6 are referred to as assume-guarantee contracts and guarantees are used to express relevant properties that a component should satisfy, e.g. a safety or a security property. As a notational convention, the indices of a contract, the corresponding set of assumptions, and the corresponding guarantee always match, e.g. $K_{j}=\left(\mathcal{A}_{j}, G_{j}\right)$, while a particular assumption within $\mathcal{A}_{j}$ is denoted $A_{i}^{j}$.

Some CBD frameworks consider contracts also to be specifications. Here, we distinguish between them because a specification does not necessarily correspond to the assume-guarantee relation specified by a contract.

The idea of separating the responsibilities between a component and its environment can be best seen from the following definition, where the notation $C \triangleright \mathcal{A}_{j}$ is a shorthand for $C \triangleright A_{1}^{j} \sqcap \cdots \sqcap A_{n}^{j}$ where each $A_{i}^{j} \in \mathcal{A}_{j}$.

Definition 7 (Satisfy Contract). Component $C$ satisfies a contract $\left(\mathcal{A}_{j}, G_{j}\right)$, denoted $C \triangleright\left(\mathcal{A}_{j}, G_{j}\right)$, if

$$
\forall C_{e} . C_{e} \triangleright \mathcal{A}_{j} \rightarrow C_{e} \otimes C \triangleright G_{j}
$$

Less formally, given a contract $\left(\mathcal{A}_{j}, G_{j}\right)$, a component $C$ can be developed independently of other components, with respect to the contract. Then, whenever component $C$ is composed with a component $C_{e}$, often referred as the environment of $C$, which implements each assumption in $\mathcal{A}_{j}$, then the composition of $C$ and $C_{e}$ implements $G_{j}$. To express that a component $C$ should satisfy a contract $K$, relation allocated to is introduced, denoted allTo $(K, C)$.

Finally, it should be noted that the presented CBD framework assumes that the specification satisfaction is monotonic with respect to component composition. This means that if a component $C_{1}$ satisfies a specification $S$, once it is composed with another component $C_{2}$, their composition still satisfies $S$. Although monotonicity does not hold in some cases [83], the remainder of the paper assumes monotonicity because capturing non-monotonic cases only extends but does not conflict with any of the presented CBD concepts.

\section{Specification Structure}

With the goal to obtain a holistic, and syntactic model of a system, this section introduces the specification structure, which contains all the contracts, their relations, and the components of a system. A similar model, called a contractstructure, has been presented previously [81] but it contained only the guarantees of contracts. In the following definition the notation $S_{1} \equiv S_{2}$ means that $S_{1}$ 
and $S_{2}$ are the same specification, and a graph without loops is a graph that does not contain edges which start and end with the same node [70, p. 594].

Definition 8 (Specification structure). Let $\mathscr{A}$ be an architecture with the root component $C_{r}$. Let $\mathcal{S}$ be a finite, non-empty set of specifications that form the set of contracts $\mathcal{K}$, where each $K \in \mathcal{K}$ is allocated to at least one component from $\mathscr{A}$. Then, a specification structure $\mathfrak{D}$ for $\mathcal{S}$ is a tuple $\mathfrak{D}=(\mathcal{N}, \mathcal{E}, \mathscr{A})$, where

i) each node $n \in \mathcal{N}$ represents a single specification $S \in \mathcal{S}$, and each $S \in \mathcal{S}$ is represented by a single $n \in \mathcal{N}$,

ii) each edge $e \in \mathcal{E}$ represents a single refines or assumption of relation, denoted $e^{\mathrm{r}}=\left(S_{1}, S_{2}\right)$ and $e^{\mathrm{a}}=\left(S_{1}, S_{2}\right)$,

iii) the graph formed by $\mathcal{N}$ and $\mathcal{E}$ does not contain loops,

iv) for each $e^{\mathrm{a}}=\left(S_{1}, S_{2}\right)$, there exists a contract $\left(\mathcal{A}_{j}, G_{j}\right) \in \mathcal{K}$ such that $S_{1} \in \mathcal{A}_{j}$ and $S_{2} \equiv G_{j}$

v) for each $e^{\mathrm{r}}=\left(S, A_{i}^{j}\right)$, where $A_{i}^{j}$ is an assumption of a contract allocated to $C_{m} \in \mathscr{A}$, it holds that

a) if $S \equiv A_{i}^{k}$ of a contract $K_{k} \in \mathcal{K}$, then there exists a $C_{p} \in \mathscr{A}$ such that $C_{p}$ is the parent of $C_{m}$ and allTo $\left(K_{k}, C_{p}\right)$,

b) if $S \equiv G_{k}$ of a contract $K_{k} \in \mathcal{K}$, then there exist $C_{p} \in \mathscr{A}$ such that $C_{m}$ and $C_{p}$ are siblings and allTo $\left(K_{k}, C_{p}\right)$.

vi) for each $e^{\mathrm{r}}=\left(S, G_{j}\right)$, where $G_{j}$ is a guarantee of a contract allocated to $C_{m} \in \mathscr{A}$, it holds that $S \equiv G_{k}$ of a contract $K_{k} \in \mathcal{K}$ and there exists a $C_{p} \in \mathscr{A}$ such that $C_{m}$ is the parent of $C_{p}$, and allTo $\left(K_{k}, C_{p}\right)$.

To provide intuition for the various conditions of Def. 8, Figure 5.3a shows a visualization of a toy specification structure. The corresponding architecture, shown in Figure 5.3b contains the composite component $\mathrm{C}_{\text {sys }}$, which is composed of atomic components $\mathrm{C}_{1}-\mathrm{C}_{3}$. The specification structure in Figure $5.3 \mathrm{a}$ is formed for specifications $A_{1}-A_{4}$ and $G_{1}-G_{4}$, which in turn form contracts $\left(\left\{A_{1}\right\}, G_{1}\right)$, $\left(\emptyset, G_{2}\right),\left(\left\{A_{2}, A_{3}\right\}, G_{3}\right)$, and $\left(\left\{A_{4}, A_{2}\right\}, G_{4}\right)$. As can be seen, each specification is a node in a graph (condition (i)), and for each contract there exists an edge labeled a, to represent the assumption of relation between each assumption and the corresponding guarantee (condition (ii) and (iv)). Furthermore, the remaining edges are labeled $r$, to represent the intended refinement between pairs of 
specifications (condition (ii)). Note that for readability reasons, the graphical representation in Figure 5.3a contains multiple nodes per specification, e.g. two nodes labeled $\mathrm{A}_{2}$, but formally, Def. 8 i) allows a single node per specification and vice-versa.

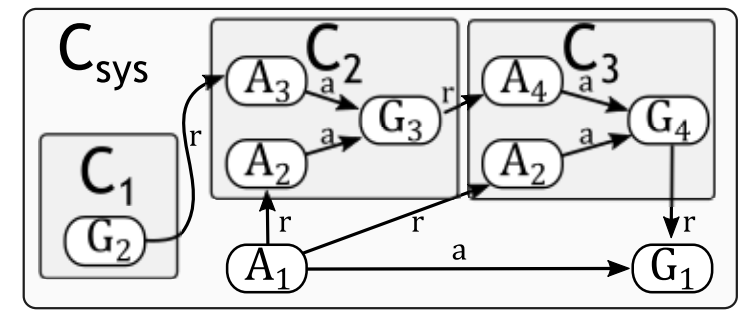

(a)

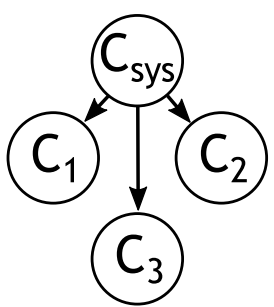

(b)

Figure 5.3: Graphical representation of a toy specification structure (a), and the corresponding architecture (b).

While conditions (i)-(iv) establish the correspondence between specifications, contracts, specification-relations and the graph of a specification structure, conditions (v) and (vi) are different in nature. They define constraints on the edges, and consequently on the relations that these edges represent. First, note that the allocated to relation is visualized by overlaying a contract over a component, e.g. in Figure $5.3 \mathrm{a}$ it holds that allTo $\left(\left(\left\{\mathrm{A}_{1}\right\}, \mathrm{G}_{1}\right), \mathrm{C}_{\mathrm{sys}}\right)$. Condition $(\mathrm{v})$ states that if a contract is allocated to a component, e.g. allTo $\left(\left(\left\{A_{2}, A_{3}\right\}, G_{3}\right), C_{2}\right)$, then the assumptions can be refined either by a guarantee of a contract allocated to a sibling component, e.g. $\mathrm{G}_{2}$, or by an assumption of contract allocated to the parent component, e.g. $A_{1}$. Conceptually speaking, if a component assumes some functionality, then this functionality is either implemented by a component of the same system (condition (v)-(b)), or the whole system assumes this functionality from some other system (condition (v)-(a)). Condition (vi) states that if a contract is allocated to a composite component, e.g. allTo $\left(\left(\left\{A_{1}\right\}, G_{1}\right), C_{\text {sys }}\right)$, then the guarantee of that contract can be refined only by a guarantee of a contract allocated to a subcomponent of the composite component, e.g. $\mathbf{G}_{4}$. Conceptually, this means that any functionality implemented by a composite component, e.g. a system, must be a consequence of the functionality implemented by its constituent components.

Note that within a particular company, artifacts such as different types of components and specifications reside in different tools, and are expressed in mul- 
tiple notations. The purpose of a specification structure is to be an integrated representation of all these artifacts and not a replacement. This representation should be constructed on-demand for analysis purposes, and although possible, it is not intended to be used as a concrete modeling-framework in everyday engineering. When it comes to the possible analyses, for example, the specification structure matches well with the principles from several functional safety standards [48, 76]. Namely, they require the decomposition of higher level requirements into lower level requirements, e.g. refinement of $\mathrm{G}_{1}$ into $\mathrm{G}_{4}$, in conjunction with the decomposition of the system architecture, e.g. $\mathrm{C}_{\text {sys }}$ is composed of $\mathrm{C}_{3}$. The existence of such formal, integrated model of a system can then be used to analyze the compliance with the requirements of such standards.

While Def. 8 defines the possible nodes and edges within a specification structure, it does not impose syntactic constraints that prevent incomplete, or inconsistent specification structures. For example, there could exist assumptions or guarantees that are not intended to be refined by any specification, and consequently not satisfied by any component. For example, in Figure 5.3, if the intended refinement between $\mathrm{G}_{2}$ and $\mathrm{A}_{3}$ would not exist, then such model would represent a system where $\left(\left\{A_{3}, A_{2}\right\}, G_{3}\right)$ is not satisfied according to Def. 7. Also, the graph of the specification structure, and consequently the specifications within the contracts, might contain directed cycles which can lead to a flawed system implementation. To avoid such cases, additional constraints are introduced.

Definition 9 (Proper specification structure). A specification structure $\mathfrak{D}$ is proper if

i) for each $A_{i}^{j}$ of a contract allocated to a non-root component $C_{m} \in \mathscr{A}$, there exists an $S \in \mathcal{S}$ and an edge $e^{\mathrm{r}}=\left(S, A_{i}^{j}\right)$ such that either

a) $S \equiv A_{l}^{k}$ of a contract $K_{k} \in \mathcal{K}$ and there exists a $C_{p} \in \mathscr{A}$ such that $C_{p}$ is the parent of $C_{m}$ and allTo $\left(K_{k}, C_{p}\right)$ or,

b) $S \equiv G_{k}$ of a contract $K_{k} \in \mathcal{K}$ and there exists a $C_{p} \in \mathscr{A}$ such that $C_{p}$ and $C_{m}$ are siblings and allTo $\left(K_{k}, C_{p}\right)$.

ii) for each $G_{j}$ of a contract allocated to a non-leaf component $C_{m} \in \mathscr{A}$, there exits an edge $e^{\mathrm{r}}=\left(G_{k}, G_{j}\right)$ and a component $C_{p} \in \mathscr{A}$, such that $G_{k}$ is the guarantee of a contract $K_{k} \in \mathcal{K}, C_{m}$ is the parent of $C_{p}$, and $\operatorname{allTo}\left(K_{k}, C_{p}\right)$

iii) $\mathfrak{D}$ does not contain a directed cycle. 
Assuming that component composition is monotonic [83, the following theorem is the key idea of CBD and it corresponds to the dominance relation [35].

Theorem 1. Let $\mathscr{A}$ be an architecture with the root $C_{r}$, and let $\mathcal{K}$ be a set of contracts such that each $K \in \mathcal{K}$ is allocated to at least one component from $\mathscr{A}$ and contract $K_{r} \in \mathcal{K}$ is allocated to $C_{r}$. Let $\mathcal{K}_{A t} \subseteq \mathcal{K}$ be the set of contracts allocated to atomic components $\mathcal{C}_{A t} \subseteq \mathscr{A}$. If

i) $\forall K \in \mathcal{K}_{A t} \cdot \forall C \in \mathcal{C}_{A t} \cdot \operatorname{allTo}(K, C) \rightarrow C \triangleright K$,

ii) $\mathcal{K}$ forms a proper specification structure $\mathfrak{D}$,

iii) for each edge $e^{\mathrm{r}}=\left(S_{1}, S_{2}\right)$, it holds that $S_{1} \sqsubseteq S_{2}$,

then it holds that $C_{r} \backslash K_{r}$.

Proof. The proof can be found in Appendix 1

Theorem 1 essentially states that: (i) if the atomic components satisfy the allocated contracts, e.g. software and hardware components, and (ii) if the system design follows the prescribed design principles, here captured as a proper specification structure, and (iii) if the intended refinement specified by $e^{\mathrm{r}}$ edges actually corresponds to refinement, then it can be inferred that the overall system satisfies the allocated contract.

The theory presented so far does not support specifying systems, i.e. composite components, that are configurable. In the next section, the presented CBD framework is extended with product line constructs.

\section{Product-Line Extension of the CBD Frame- work}

This section presents the first contribution, which is a product-line extension of the theory presented in Section 3.2 and Section 4 . Before the formal definitions, we provide some intuition.

Consider using the presented CBD framework to represent and analyze a configurable system. This implies that the design of each system variant would have to be defined as a separate specification structure $\mathfrak{D}$. The upper part of Figure 5.4 shows two specification structures of the composite component $\mathrm{C}_{\text {sys }}$ for configurations $\gamma_{1}$ and $\gamma_{2}$, which represent two different system variants. For different configurations, $\mathrm{C}_{\mathrm{sys}}$ is composed of different components, 
i.e. $\mathrm{C}_{\mathrm{sys}}=\mathrm{C}_{1} \otimes \mathrm{C}_{2}$ versus $\mathrm{C}_{\mathrm{sys}}=\mathrm{C}_{1} \otimes \mathrm{C}_{3}$, and different contracts are allocated to the same components, e.g. allTo $\left(\left(\left\{A_{3}\right\}, G_{3}\right), C_{1}\right)$ versus allTo $\left(\left(\left\{A_{4}\right\}, G_{3}\right), C_{1}\right)$. Besides the fact that common components and contracts would be duplicated across multiple specification structures, more importantly, the reasoning about whether each variant of $C_{\text {sys }}$ satisfies the contract $\left(\left\{\mathrm{A}_{1}\right\}, \mathrm{G}_{1}\right)$, would have to be performed per variant. Because the number of configurations, and consequently system variants can be very high, performing per-variant analysis is usually not feasible. The alternative is the approach proposed in the present paper, namely to directly create a product-line specification structure and analyze it simultaneously for all configurations, regardless of their number. Such model would correspond to the integrated platform from which variants of the system are derived. The bottom part of Figure 5.4 shows the product-line specification structure that simultaneously expresses the two variants of $C_{\text {sys }}$.

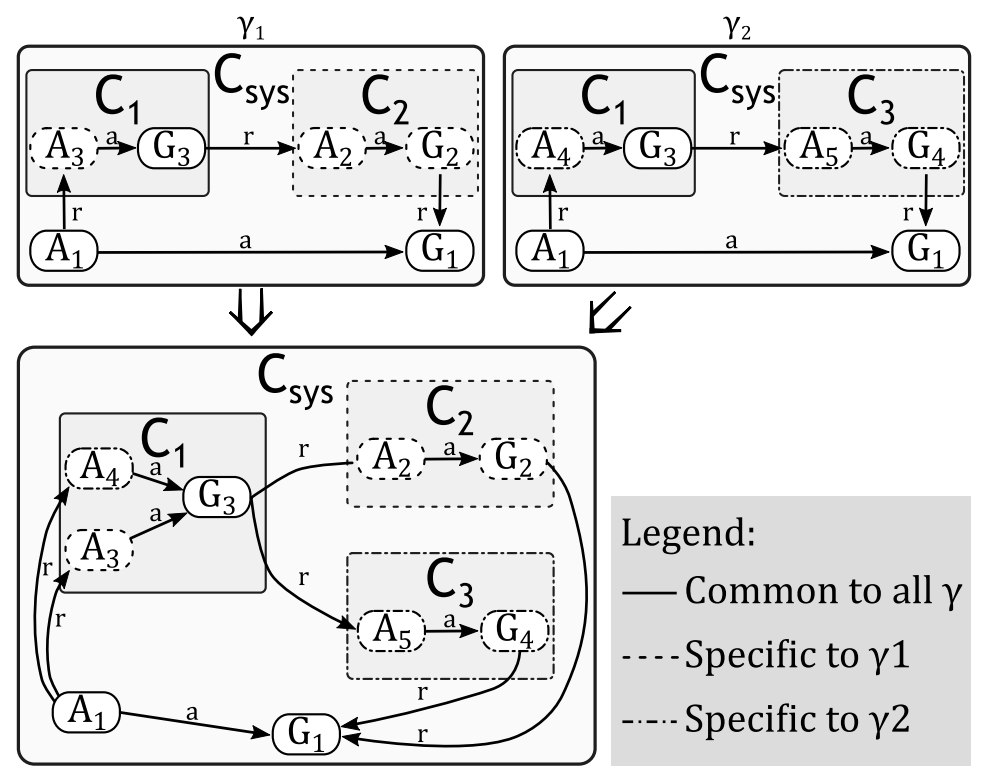

Figure 5.4: Per-configuration specification structures (top), and the concept of a product-line specification structure (bottom).

Because a product-line specification structure represents multiple variants, the idea is that if each variant is a proper specification structure, then Theorem 1 can be used to infer that the configurable system, e.g. $\mathrm{C}_{\text {sys }}$ in Figure 5.4, satisfies 
the contract allocated to it, for all configurations $\gamma$. To ensure that the design of each variant is a proper specification structure, and because a product-line specification structure represents a configurable system, additional constraints must be enforced. In Figure 5.4 guarantee $G_{3}$ is intended to refine assumption $A_{2}$, and component $C_{1}$ is intended to satisfy the contract $\left(\left\{A_{3}\right\}, G_{3}\right)$. Given a configuration $\gamma$, to which guarantee $A_{2}$ applies, it must be ensured that each of the artifacts $C_{1}, A_{3}$, and $G_{3}$ also apply to configuration $\gamma$. Also, either assumption $\mathrm{A}_{3}$ or assumption $\mathrm{A}_{4}$ should apply to configuration $\gamma$, but not both of them.

To understand the necessity for enforcing additional constraints, consider the first case. If there exists a variant that contains artifacts $C_{2}, A_{2}, G_{2}$, and $G_{1}$ but not all of them, that could mean that either: (i) the component $C_{2}$ is part of the variant but the contract $\left(A_{2}, G_{2}\right)$ does not, or (ii) $A_{2}$ and $G_{2}$ do not jointly comprise the variant, i.e. the contract $\left(A_{2}, G_{2}\right)$ is ill-formed with respect to Def. 8. The described issues are referred to as configuration mismatches [25], and in general they represent a mismatch between the product line design, here represented as a CBD model, and the product line specification, which is here represented as a variability model.

Besides verifying the absence of configurations mismatches, it is crucial that such verification is performed only once, against the product-line specification structure, and not against parts of the product-line specification structure that represent a particular variant. In this way, the challenge of analyzing billions of variants is avoided. In the following two subsections, the product-line specification structure is formally defined, presence conditions are introduced, and the constraints that ensure absence of configuration mismatches are defined.

\subsection{CBD Model of a Configurable System}

The definition of a product-line specification structure is similar to specification structure from Def. 8, with the difference that it simultaneously represents the design of several configurations from $\Gamma$. Before formally introducing the product-line specification structure, we define the product-line architecture where components can be shared between different system variants, i.e. the architecture is not a tree as in Def. 4.

Definition 10 (PL Architecture). A PL architecture $\widehat{\mathscr{A}}$ is a finite, non-empty set of components organized into a rooted, directed, acyclic graph where each leaf node is an atomic component, and each non-leaf node $C$ with children nodes $C_{1}, \ldots, C_{n}$ is a composite component, such that $C=\bigotimes_{i=1}^{n} C_{n}$.

To create a product-line specification structure that represents the design of 
all configurations from $\Gamma$, regardless of their number, it is necessary to define to which configurations from $\Gamma$ each component and each specification apply to. As discussed above, this is achieved by labeling each specification and each component with a presence condition.

Definition 11 (PL Specification Structure). Let $\widehat{\mathscr{A}}$ be a PL architecture with the root component $C_{r}$. Let $\mathcal{S}$ be a finite, non-empty set of specifications that form the set of contracts $\mathcal{K}$ where each $S \in \mathcal{S}$ is either an assumption or a guarantee of a contract $K \in \mathcal{K}$, and each $K \in \mathcal{K}$ is allocated to at least one component from $\widehat{\mathscr{A}}$. Then, a PL specification structure $\widehat{\mathfrak{D}}$ for $\mathcal{S}$ is a tuple $\widehat{\mathfrak{D}}=(\mathcal{N}, \mathcal{E}, \widehat{\mathscr{A}}, p)$, where

i) conditions $i$ ) vi) from Def. 8 hold,

ii) $p$ is a function that labels each specification $S \in \mathcal{S}$, and each component $C \in \widehat{\mathscr{A}}$, with a presence condition $\varphi$ according to Def. 3 .

Similarly to the basic CBD framework, we also define a proper PL specification structure.

Definition 12 (Proper PL specification structure). A PL specification structure $\widehat{\mathfrak{D}}$ is proper if

i) condition i) of Def. 9 holds,

ii) condition ii) of Def. 9 holds.

Unlike Figure 5.4 which just provides the intuition for a PL specification structure, Figure 5.5 shows a proper PL specification structure, the underlying architecture, and examples of presence conditions, for a realistic configurable system described by the feature model from Figure $5.2 \mathrm{~b}$

The example represents the design of a toy product-line where $\mathrm{C}_{\mathrm{sys}}$ is composed of $\mathrm{C}_{\text {subsys1 }}$ and $\mathrm{C}_{\text {subsys2 } 2 \text {. The meaning of the visual elements is identical }}$ as for Def. 8 with the additional visualization of presence conditions that label components and specifications (condition (ii) of Def. 11). The presence conditions are written with respect to the feature model from Figure 5.2 , where the symbol $\mathrm{T}$ denotes that the presence condition is always true, i.e. components and specifications labeled with $\mathrm{T}$ apply to all configurations from $\Gamma$. Each of the remaining presence conditions defines a subset of $\Gamma$. For example, presence conditions $\varphi_{s y s}, \varphi_{1}$, and $\varphi_{2}$ define that $\mathrm{C}_{\text {sys }}$ is composed of $\mathrm{C}_{\text {subsys } 1}$ for all configurations where $f_{4}=$ true, composed of $\mathrm{C}_{\text {subsys2 }}$ for all configurations 


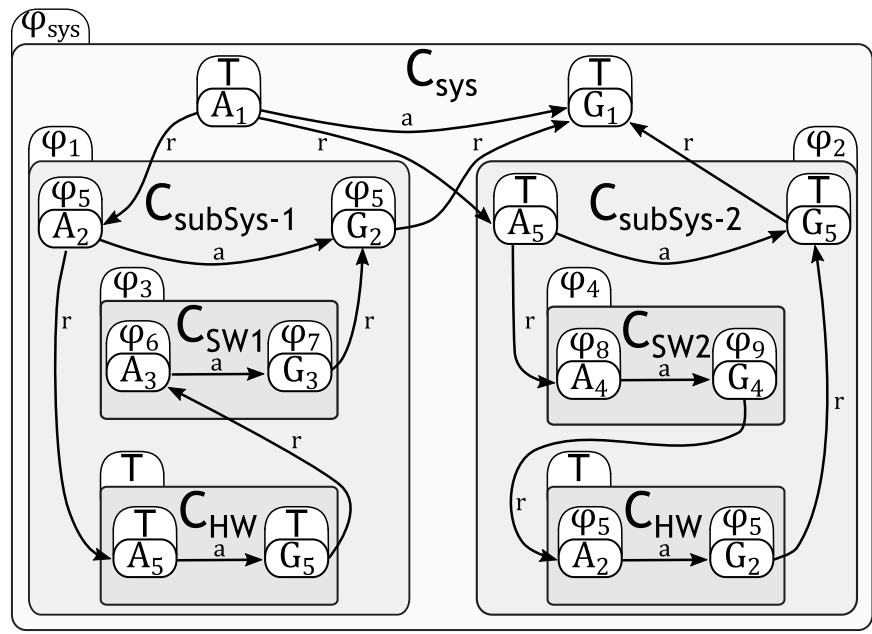

(a)

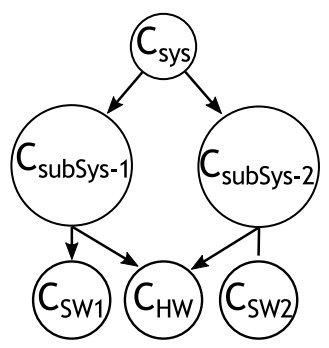

(b)

$$
\begin{aligned}
& \varphi_{\text {sys }}=\mathrm{f}_{4} \vee \mathrm{f}_{5}, \\
& \varphi_{1}=\mathrm{f}_{4}, \\
& \varphi_{2}=\mathrm{f}_{5}, \\
& \varphi_{5}=\mathrm{f}_{8},
\end{aligned}
$$

(c)

Figure 5.5: Example proper PL specification structure (a), the corresponding $\mathrm{PL}$ architecture (b), and example presence conditions (c).

where $f_{5}=$ true, and because $f_{4}$ and $f_{5}$ form an alternative group in the feature model, no valid configuration will simultaneously assign the value true to both $f_{4}$ and $f_{5}$. This means that $\mathrm{C}_{\text {sys }}$ exists in two different variants with respect to component composition.

According to Def. 10, and in contrast to Def. 4, a single component can be a part of multiple compositions, e.g. $\mathrm{C}_{\text {subsys1 }}$ and $\mathrm{C}_{\text {subsys2 }}$ execute different software, but they have a common hardware, namely $\mathrm{C}_{\mathrm{HW}}$. Also, because a proper PL specification structure is a superimposition of several configurationspecific specification structures, in the general case, the graph of $\widehat{\mathfrak{D}}$ can contain a directed cycle and therefore the condition iii) from Def. 9 is not included in Def. 12. For example, in Figure 5.5. contract $\left(A_{5}, G_{5}\right)$ is allocated to $\mathrm{C}_{\mathrm{HW}}$ and $\mathrm{C}_{\text {subsys2 }}$. Although these are visually represented separately for comprehension, formally the set of edges $\mathcal{E}$ of the proper PL specification structure in Figure 5.5 contains the cycle $e^{\mathrm{r}}=\left(A_{2}, A_{5}\right), e^{\mathrm{r}}=\left(A_{5}, A_{4}\right), e^{\mathrm{a}}=\left(A_{4}, G_{4}\right), e^{\mathrm{r}}=\left(G_{4}, A_{2}\right)$.

As outlined in Section 3.1 artifacts are labeled with presence conditions to define the set of configurations to which the artifacts apply. Then, function eval evaluates the presence conditions for a given configuration, and the artifacts whose presence conditions evaluate to true are selected, thus obtaining the 
artifacts that comprise the variant that corresponds to the selected configuration. To be able reason about this process, we formally introduce the concept of a variant which is derived from a PL specification structure by removing the artifacts whose presence conditions evaluate to false for a given configuration.

Definition 13 (Variant). Let $\widehat{\mathfrak{D}}$ be PL specification structure, and let $\gamma \in \Gamma$ be a configuration. A variant, denoted $\mathfrak{D}_{\gamma}$, is a tuple $\mathfrak{D}_{\gamma}=\left(\mathcal{N}_{\gamma}, \mathcal{E}_{\gamma}, \mathscr{A}_{\gamma}\right)$ where

i) $\mathcal{N}_{\gamma} \subseteq \mathcal{N}$ such that $\forall n \in \mathcal{N}_{\gamma}$ where $n$ represents a specification $S$, it holds that $\operatorname{eval}(p(S), \gamma)=$ true,

ii) $\mathcal{E}_{\gamma} \subseteq \mathcal{E}$, where for each $e=\left(S_{1}, S_{2}\right)$ it holds that $\operatorname{eval}\left(p\left(S_{1}\right), \gamma\right)=\operatorname{eval}\left(p\left(S_{2}\right), \gamma\right)$ $=$ true,

iii) $\mathscr{A}_{\gamma} \subseteq \widehat{\mathscr{A}}$ such that $\forall C \in \mathscr{A}_{\gamma} \cdot \operatorname{eval}(p(C), \gamma)=$ true,

iv) components from $\mathscr{A}_{\gamma}$ form a tree.

A variant $\mathfrak{D}_{\gamma}$ is not necessarily a specification structure according to Def. 8 . For example, in the context of Figure 5.5, it might be the case that $\operatorname{eval}\left(\varphi_{6}, \gamma\right)=$ $\operatorname{eval}\left(\varphi_{7}, \gamma\right)=\operatorname{true}$ and $\operatorname{eval}\left(\varphi_{3}, \gamma\right)=$ false, i.e. for configuration $\gamma$ contract $\left(A_{3}, G_{3}\right)$ is not allocated to any component. On the other hand, if we could verify that any variant is a specification structure (c.f. Def. 8), and furthermore a proper specification structure (c.f. Def. 9), then Theorem 1 could be extended to claim that for any configuration, a configurable system satisfies the contracts allocated to it.

\subsection{Constraints on Presence Conditions}

To ensure that each variant is a proper specification structure according to Def. 9, we introduce the following conditions, and we refer to them as invariance with respect to configurations, hereinafter only invariant. In the following definitions, the symbol $\models_{\Theta}$ represents logical entailment with respect to the set of Boolean formulas $\Theta$ of a variability model $\mathfrak{M}$. More formally, $f_{i} \models_{\Theta} f_{j}$ is equivalent to $f_{i}, \Theta \models f_{j}$. For example, given Boolean features $f_{1}$ and $f_{2}$ it holds that $f_{1} \not \models f_{2}$ but in the context of the feature model from Figure 5.2a, where $\Theta$ contains formula $f_{1} \rightarrow f_{2}$, then it holds that $f_{1} \models_{\Theta} f_{2}$.

Definition 14 (Invariant Contract). A contract $\left(\mathcal{A}_{j}, G_{j}\right)$ is invariant if

i) $\forall A_{i}^{j} \in \mathcal{A}_{j} \cdot p\left(A_{i}^{j}\right) \models_{\Theta} p\left(G_{j}\right)$, 
ii) $\forall A_{i}^{j} \in \mathcal{A}_{j} \cdot p\left(G_{j}\right) \models_{\Theta} p\left(A_{i}^{j}\right)$.

Less formally, Def. 14 defines the conditions under which each contract is well-formed according to Def. 6 for each configuration.

Definition 15 (Invariant Allocation). An allocation of a contract $\left(\mathcal{A}_{j}, G_{j}\right)$ to possibly $n$ different components, is invariant if $p\left(G_{j}\right) \models_{\Theta} \bigvee_{i=1}^{n} p\left(C_{i}\right)$.

The condition of Def. 15 ensures that if a contract is a part of a variant, then at least one component to which the contract is allocated to, is also a part of the same variant. Note that Def. 15 includes two cases of invariance. The first case is if multiple components are redundant and should satisfy the same contract. In that case, for any $\gamma$, presence conditions of multiple components $C_{i}$ from Def. 15 will evaluate to true whenever $p\left(G_{j}\right)=$ true. The second case is if different components should satisfy the same contract for different configurations. In that case, for different $\gamma$, the presence condition of a single component $C_{i}$ from Def. 15 will evaluate to true whenever $p\left(G_{j}\right)=$ true.

Definition 16 (Invariant Composition). Composition of $n$ components $C_{1}, \ldots, C_{n}$, into $C=\bigotimes_{i=1}^{n} C_{i}$ is invariant if it holds that $\forall C_{i} \in\left\{C_{1}, \ldots C_{n}\right\} . p\left(C_{i}\right) \models_{\Theta}$ $p(C)$.

The purpose of Def. 16 is to ensure that if a component is a part of a variant, then its parent component is also a part of the same variant. For example, if the presence condition of $\mathrm{C}_{\mathrm{SW} 1}$ evaluates to true, then the presence condition of $\mathrm{C}_{\text {subsys1 }}$ should evaluate to true.

Given a PL specification structure $\widehat{\mathfrak{D}}$ with invariant contracts, allocation, and composition, instantiating $\widehat{\mathfrak{D}}$ for an arbitrary configuration $\gamma$, results in a specification structure according to Def. 8 .

Theorem 2. Let $\Gamma$ be a set of valid configurations, and let $\widehat{\mathfrak{D}}$ be a proper $P L$ specification structure. If

i) each contract is invariant,

ii) allocation of contracts to components from $\widehat{\mathscr{A}}$ is invariant,

iii) composition of child into parent components in $\widehat{\mathscr{A}}$ is invariant,

then each variant $\mathfrak{D}_{\gamma}$, is a specification structure.

Proof. The proof can be found in Appendix 1 
Theorem 2 establishes the conditions under which a variant is a specification structure. However, using Theorem 1 requires that a variant is a proper specification structure. The following theorem defines additional conditions under which each variant is a proper specification structure according to Def. 9. Note that one of the conditions is that the graph of $\widehat{\mathfrak{D}}$ does not contain a directed cycle, and consequently, no variant $\mathfrak{D}_{\gamma}$ contains a directed cycle as required by Def. 9. Although the cycles such as the one described in Figure 5.5 can be avoided, e.g. by allowing that a particular contract can be allocated only to components at the same architectural level, in the general case, detecting cycles may require the exploration of the complete graph of $\widehat{\mathfrak{D}}$, which can be done efficiently by using known algorithms, e.g. depth-first search [70, p.787].

Theorem 3. Let $\Gamma$ be a set of valid configurations, and let $\widehat{\mathfrak{D}}$ be a proper $P L$ specification structure such that each variant $\mathfrak{D}_{\gamma}$ is a specification structure. If

i) for each $A_{i}^{j} \in \mathcal{S}$, and edges $e^{\mathrm{r}}=\left(S_{1}, A_{i}^{j}\right), \ldots,\left(S_{n}, A_{i}^{j}\right)$ it holds that $p\left(A_{i}^{j}\right) \models_{\Theta} \bigvee_{k=1}^{n} p\left(S_{k}\right)$,

ii) for each $G_{j} \in \mathcal{S}$, and edges $e^{\mathrm{r}}=\left(G_{1}, G_{j}\right), \ldots,\left(G_{n}, G_{j}\right)$ it holds that $p\left(G_{j}\right) \models_{\Theta} \bigvee_{k=1}^{n} p\left(G_{k}\right)$

iii) $\widehat{\mathfrak{D}}$ does not contain a directed cycle,

then each $\mathfrak{D}_{\gamma}$ is proper specification structure.

Proof. The proof can be found in Appendix 1.

A direct consequence of Theorem 2 and Theorem 3 in conjunction with Theorem 1 is the following corollary.

Corollary 1. Let $\widehat{\mathfrak{D}}$ be a proper PL specification structure for a set of specification $\mathcal{S}$ that form a set contracts $\mathcal{K}$. Let $K_{r} \in \mathcal{K}$ be the contract allocated to the root component $C_{r} \in \widehat{\mathscr{A}}$, and let $\mathcal{K}_{A t} \subseteq \mathcal{K}$ denote the set of contracts allocated to the set of atomic components $\mathcal{C}_{A t} \subseteq \widehat{\mathscr{A}}$. Let $\Gamma$ be a set of valid configurations, defined by a variability model $\mathfrak{M}$. If

i) premises of Theorem 2 hold,

ii) premises of Theorem 3 hold,

iii) for each edge $e^{\mathrm{r}}=\left(S_{1}, S_{2}\right)$, it holds that $S_{1} \sqsubseteq S_{2}$,

iv) $\forall K \in \mathcal{K}_{A t} . \forall C \in \mathcal{C}_{A t} \cdot \operatorname{allTo}(K, C) \rightarrow C \triangleright K$, 
then it holds that $C_{r} \triangleright K_{r}$ for each $\gamma \in \Gamma$.

Corollary 1 summarizes the main results so far. If a proper PL specification structure is considered to be a representation of a product line, i.e. the integrated platform from which variants are derived, then conditions (i)-(iii) of Corollary 1 must be satisfied by the design, and condition (iv) must be satisfied by the implementation, in order to be able to claim that for configuration the contract $K_{r}$ is satisfied. As will be shown in Section 7, providing evidence that the premises of Corollary 1 hold is the basis for creating assurance-case arguments.

However, before proceeding to the creation of assurance-case arguments, the applicability of the CBD framework to a realistic setting is evaluated.

\section{Applying the CBD Framework to a Real Prod- uct Line}

This section presents an application of the extended CBD framework with the objective to understand if it can be used to represent and analyze a real, safetycritical product-line. The hypothesis is that applying the CBD framework to a real product-line will either lead to the creation of proper PL specification structure, thus enabling the application of Corollary 1] or one or more conditions of the CBD framework will not be satisfied, and this might lead to recommendations to improve the product-line design or implementation. Recall that the purpose of the CBD framework is not to be used as a concrete modelingframework for different artifacts within the product line. Consequently, we do not attempt to evaluate if it is possible, and what is the required effort, to adopt the CBD framework in everyday engineering. Rather, a CBD model is a formal representation that is constructed on-demand for analysis purposes, i.e. the CBD framework is intended to enable preferably automated analysis of arbitrary product lines, which are developed using tools and notations that best suite everyday activities within a particular company.

The questions that this section seeks to answer are:

Q1 Given a real product line with a variety of concrete artifacts in different notations, is it possible to represent such product line as a CBD model?

Q2 If the CBD representation of a product line violates some conditions of the CBD framework, can these violations be translated into actionable engineering insights, or are these a consequence of formalization of the CBD-framework? 


\subsection{Methodology}

Given the open-ended nature of the above questions, we adopt the structure of an exploratory case study [72] to reach the answers. In accordance with [72, the phases of the performed case study are shown Figure 5.6. The product line used as the case is a real, safety-critical product line from the heavy vehicle manufacturer SCANIA.

Selecting the Case. The selected case is the Fuel-Level Display (FLD) system, whose functionality is available in each ScAnIA vehicle. This system was selected for several reasons. Firstly, it is a typical system from the automotive domain. It is implemented across several Electronic Control Units (ECUs) within the vehicle, its behavior directly depends on the configuration selected by the customer, and the artifacts within the product-line are created in a V-like development process. Also, the system is safety-critical (internal SCANIA rating corresponds to ASIL B), which means that the artifacts should be of sufficient quality for a rigorous analysis. Last, but not the least, a practical consideration is that parts of the FLD system have been published earlier, thus ScANIA was willing to allow a publication based on the FLD system. It should be noted that the analyzed version of the FLD system, with the corresponding artifacts, was developed around 2015 .

Selecting the Unit of Analysis. After selecting the case, the authors collected the artifacts of the FLD system with the help of two SCANIA engineers who pointed out the location and the correct versions of the artifacts. Given the overall system, the authors selected a part of the system to be the unit of analysis [72] such that the unit is comprehensive enough to apply the CBD framework, but small enough for a manual analysis and subsequent presentation of the results. This activity has revealed ambiguities within the analyzed artifacts, primarily among the natural-language requirements, and another consultation was made with the same SCANIA engineers in order to disambiguate the artifacts and also to confirm that the unit of analysis actually represents a part of the end-to-end functionality of the FLD system.

Executing the Study. The actual synthesis of the CBD model was performed without the help of the engineers because our long term vision is that a CBD model of a system can be created automatically. First, explicit entities within the collected artifacts were mapped to the concepts of the CBD framework, i.e. components, specifications, presence conditions. Then, the explicit traceability 


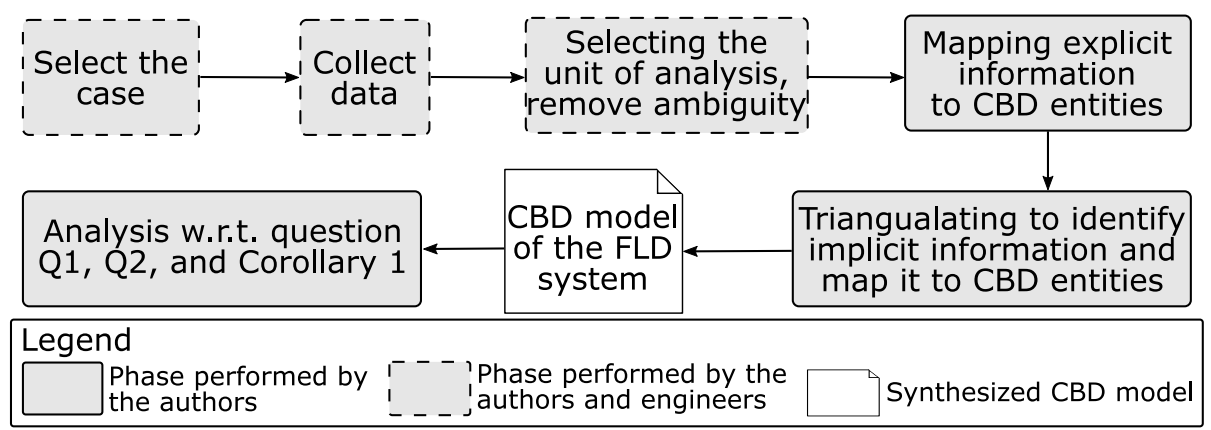

Figure 5.6: Phases of the exploratory case study.

links within the artifacts were mapped to the relations of the CBD framework, i.e. allocated to, refines, composition, assumption of. At this point, the CBD model was not even a PL specification structure because the development practice in SCANIA was to allocate requirements only to logical components, which represent the overall system, and to the main software components. Therefore, the requirements and the traceability links for the implementation components at various abstraction levels were missing. To identify the implicit information, we relied on triangulation 72 between the available artifacts, with respect to the conditions of the CBD framework. Triangulation is a frequently used method in qualitative research where the same problem is analyzed based on several datasources, by using different methods etc. Triangulation is typically performed to increase the validity of the results, and to minimize the risk of introducing bias. In the context of the present paper, triangulation was performed over several data sources to ensure the validity of inferred information. For example, descriptive parts of the available requirements documents contained informal text with implicit references to the ECUs that should implement the requirements within the documents. Therefore, this text could have been used as the source to infer the missing allocation relations. Instead, triangulation was performed to ensure the validity of the inferred information. Firstly the requirements within the documents were analyzed to identify the variables that the requirements constrain. Then, these variables would be identified in the source code of an ECU, which would lead to the software function that is producing this value. Only if the value type and range from a requirement and from the software function would match, we would infer which that this particular specification is allocated to the particular software function. 
After this step, the final CBD model was obtained, and it was analyzed with respect to the conditions of Corollary 1 to answer the formulated questions. The following sections present the FLD system in more detail, then we present the obtained CBD model, and then discuss the answers to the formulated questions in the context of lessons learned during the creation of the CBD model.

\subsection{The FLD System and the Analyzed Artifacts}

The functionality of the FLD system is twofold. Firstly, the FLD should ensure that the fuel volume displayed to the driver, corresponds to the actual fuel volume in the fuel tank. Secondly, if the fuel volume falls below a certain threshold, the FLD should display a warning to the driver. The FLD is safety-critical because if the displayed fuel volume is higher than the actual one, the driver might continue driving until unexpectedly running out of fuel. Consequently, the engine will shut-down, leading to loss of power-steering which is essential for heavy-vehicle steering.

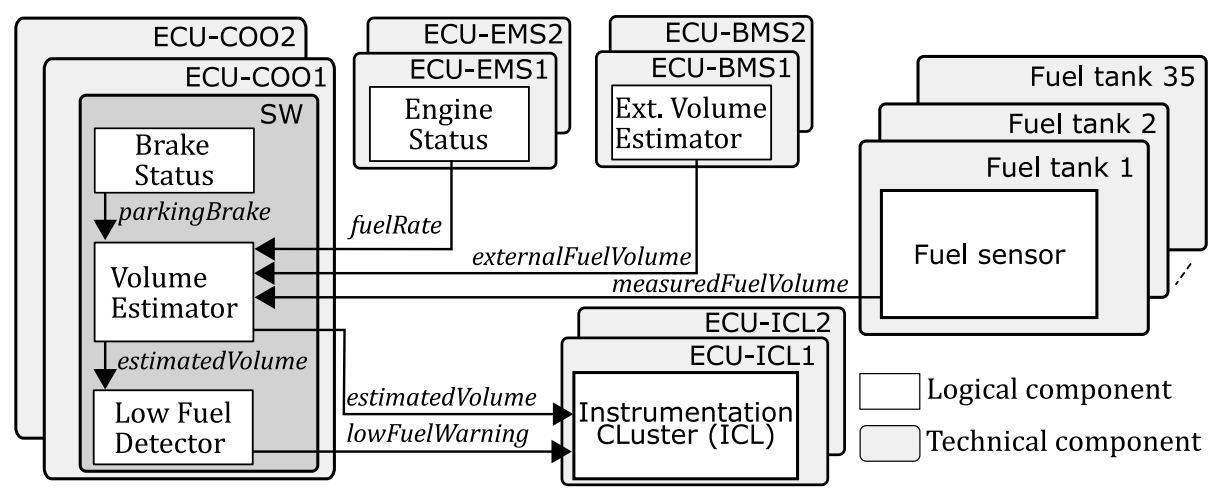

Figure 5.7: Illustration of the FLD system architecture.

Table 5.1 shows the artifacts that were analyzed in order to extract the information about the FLD system while Figure 5.7 shows the main logical and technical components of the FLD system, together with information flows between the components. The model in Figure 5.7 is created primarily from the data from the logical architecture and the product structure which contains all technical components of the FLD system. The specified behavior of the different components, as defined in analyzed requirements documents, is as follows. The 
Table 5.1: Analyzed artifacts of the FLD

\begin{tabular}{|c|c|c|}
\hline Artifact & Format & Content \\
\hline $\begin{array}{l}\text { Logical } \\
\text { architecture }\end{array}$ & $\begin{array}{l}\text { Custom } \\
\text { graphical }\end{array}$ & $\begin{array}{l}\text { Logical components with presence conditions, allo- } \\
\text { cated to ECUs, CAN signals between components }\end{array}$ \\
\hline $\begin{array}{l}\text { Requirements } \\
\text { document }\end{array}$ & $\begin{array}{l}\text { Controlled } \\
\text { natural } \\
\text { language }\end{array}$ & $\begin{array}{l}\text { Functional, functional-safety requirements with } \\
\text { presence conditions of the FLD system }\end{array}$ \\
\hline $\begin{array}{l}\text { Requirements } \\
\text { document }\end{array}$ & $\begin{array}{l}\text { Controlled } \\
\text { natural } \\
\text { language }\end{array}$ & $\begin{array}{l}\text { Per logical component, functional, functional-safety } \\
\text { requirements with presence conditions. }\end{array}$ \\
\hline Source code & C lang. & $\begin{array}{l}\text { Implementation of logical components with config- } \\
\text { uration variables }\end{array}$ \\
\hline $\begin{array}{l}\text { Configuration } \\
\text { database }\end{array}$ & $\begin{array}{l}\text { Custom } \\
\text { textual }\end{array}$ & $\begin{array}{l}\text { Presence conditions for different values of configu- } \\
\text { ration variables }\end{array}$ \\
\hline $\begin{array}{l}\text { Product } \\
\text { structure }\end{array}$ & $\begin{array}{l}\text { Custom } \\
\text { textual }\end{array}$ & $\begin{array}{l}\text { Hardware, electrical, electromechanical components } \\
\text { of the FLD system with presence conditions }\end{array}$ \\
\hline $\begin{array}{l}\text { Verification } \\
\text { documents }\end{array}$ & $\begin{array}{l}\text { Custom } \\
\text { textual }\end{array}$ & $\begin{array}{l}\text { Verification results of SW and HW components, } \\
\text { ECUs, and the FLD system }\end{array}$ \\
\hline $\begin{array}{l}\text { Variability } \\
\text { model }\end{array}$ & $\begin{array}{l}\text { Custom } \\
\text { textual }\end{array}$ & $\begin{array}{l}\text { Around } 50.000 \text { features with corresponding Boolean } \\
\text { constraints }\end{array}$ \\
\hline
\end{tabular}

component Volume Estimator estimates the current fuel-volume, given the fuellevel sensor readings, and the current rate of fuel consumption. The estimated fuel volume is an input to the Instrumentation CLuster (ICL), which indicates the estimated volume to the driver. Note that because ICL is bought from a supplier, from the SCANIA perspective this is a logical component, implemented by a whole ECU also called ICL. The estimated fuel volume is also an input to the Low Fuel Detector, which calculates if the fuel volume is below a threshold. If so, a low fuel warning is sent to the ICL. To reliably detect re-fueling, the Volume Estimator needs the information about the parking-brake status.

Although the FLD is installed in each SCANIA vehicle, the system itself is highly-configurable and this is witnessed by the fact that almost all artifacts in Table 5.1 contain presence conditions. For example, the presence condition of the overall system defines that the FLD system can build into a truck or a bus, and for buses with more than one fuel tank, the fuel volume in additional 
tanks is estimated by the component External Volume Estimator. In such cases, the component Volume Estimator merges its estimate with the external one, and calculates the total estimate. Moreover, the logical components from Figure 5.7 are allocated differently for different variants. The allocation of the logical components from Figure 5.7 applies only to variants that use liquid fuel, while for gas-powered vehicles, Volume Estimator and Low Fuel Detector are allocated to the Engine Management System (EMS).

Regarding technical components, the ECUs also differ depending on the configuration of the vehicle. For example, COO ECU can be built with a basic or an extended hardware, both bought from suppliers, thus giving rise to C001 and C002. Furthermore, there are 35 different fuel tanks, with different shapes and volume. Also, there are 16 different types of fuel-level sensors, which implement the logical component Fuel Sensor, and that can be combined with the different fuel tanks. The variability of the FLD system is also heavily reflected in the source code through the so-called configuration variables whose values are set at load-time [71] from the configuration database depending on the vehicle configuration. For example, the C-language implementation of the Volume Estimator is essentially a large switch statement where the different cases are executed depending on the values of configuration variables. Also, the invocation of whole software components, e.g. Low Fuel Detector and External Volume Estimation, is controlled by the values of configuration variables. In summary, the estimated number of unique FLD variants is around 24,000.

In relation to Figure 5.7. the unit of analysis of the FLD system includes the COO ECU, with two different hardware and a single software, two variants of the ICL ECU, namely ICL1 and ICL2, and two fuel-tank assemblies that can be installed individually or jointly, and which include four different fuel tanks and two different sensors. In total, the unit of analysis include 24 variants of the FLD system, and it includes various types of components, at different abstraction levels, that implement the basic functionality of reading the sensor value, estimating the fuel volume, and displaying it to the driver.

\subsection{Synthesis of the CBD Model}

Given the unit of analysis, the obtained CBD model of the FLD system is shown in Figure 5.8, while the corresponding specifications are shown in Table 5.2. The presence conditions of the obtained CBD model are discussed later in Section 6.3.

All components in Figure 5.8 were directly identified from the logical architecture, product structure and source code since these were organized in terms of SCANIA equivalents of CBD components. Except in the source code, component 
$C_{\text {FLD }}$ exists in each of these artifacts because it represents the FLD system. Components $\mathrm{C}_{\text {ICL1 }}, \mathrm{C}_{\text {ICL2 } 2}, \mathrm{C}_{\text {TankL }}, \mathrm{C}_{\text {TankR }}$ and all of their subcomponents were identified from the product structure. Also, $\mathrm{C}_{\mathrm{COO}}, \mathrm{C}_{\mathrm{BasicH}}$ and $\mathrm{C}_{\mathrm{ExtendHW}}$ were also identified from the product structure. Component $\mathrm{C}_{\mathrm{Coosw}}$ represents the complete source code of $\mathrm{COO}$, and components $\mathrm{C}_{\mathrm{Fue120ms}}, \mathrm{C}_{\mathrm{R} 2 \mathrm{Perc}}$, and $\mathrm{C}_{\mathrm{Est}}$ were identified from the source code (a software-architecture artifact did not exist). Note that although COO software contains many application components which run on a common infrastructure software, here we represent only the application component of the FLD system, namely the $\mathrm{C}_{\mathrm{Fuel20ms}}$, where the name indicates how often the application is executed. Also note that $\mathrm{C}_{\mathrm{Fue120ms}}$ implements the logical component Volume Estimator from Figure 5.7. Furthermore, although $\mathrm{C}_{\text {ICL1 }}$, and $\mathrm{C}_{\text {ICL2 }}$ are complete ECUs, because they are bought from suppliers, from the SCANIA perspective they are only atomic components.

When it comes to assumptions $A_{1}-A_{11}$ and guarantees $G_{1}-G_{14}$ in Table 5.2 . these are the requirements from requirements documents, obtained after the disambiguation with Scania engineers. However, according to the SCANIA practices, the analyzed requirements were defined only for the FLD system, represented by $\mathrm{C}_{\mathrm{FLD}}$ component, and for the logical components. Namely, these are Volume Estimator, implemented by $\mathrm{C}_{\text {Fuel2oms }}$, Fuel sensor implemented by $\mathrm{C}_{\mathrm{Sens} 1}$ or $\mathrm{C}_{\mathrm{Sens} 2}$, and ICL implemented by $\mathrm{C}_{\text {ICL1 } 1}$ or $\mathrm{C}_{\text {ICL2 } 2}$. Consequently, the specifications of these components were to a large extent explicitly allocated within the requirements documents.

Individual requirements (c.f. Table 5.2 primarily defined how the different components should behave, i.e. which properties should be guaranteed, thus the majority were interpreted as guarantees, namely $\mathrm{G}_{1}, \mathrm{G}_{4}-\mathrm{G}_{9}, \mathrm{G}_{11}$, and $\mathrm{G}_{14}$. For $\mathrm{G}_{1}, \mathrm{G}_{4}, \mathrm{G}_{9}, \mathrm{G}_{11}$, and $\mathrm{G}_{14}$, the requirements documents contained explicit allocation information. For guarantees $G_{5}-G_{8}$, the allocation was inferred by identifying the values that a guarantee constrains, e.g. percent fuel level in $\mathrm{G}_{7}$, and then finding the component which produces this value, e.g. the $\mathrm{C}$-function represented by $\mathrm{C}_{\mathrm{R} 2 \mathrm{Perc}}$. Because a contract contains exactly one guarantee (c.f. Def. 6), identifying the guarantees meant that the corresponding contracts are declared.

When it comes to the assumptions of contracts, few requirements from the analyzed documents were clearly marked as necessary precursors for other requirements, i.e. assumptions, and they were explicitly allocated. These assumptions are $A_{2}, A_{3} A_{9}-A_{11}$. Assumptions $A_{2}$ and $A_{3}$ were a part of the requirements document for the overall system, and they were allocated to the ECU that implements the logical component Volume Estimator, which is $\mathrm{C}_{\mathrm{coo}}$. Also, the requirements documents contained references to the data-sheets of sensors, which in turn declared the expected environment in which they operate, i.e. $A_{10}$ 


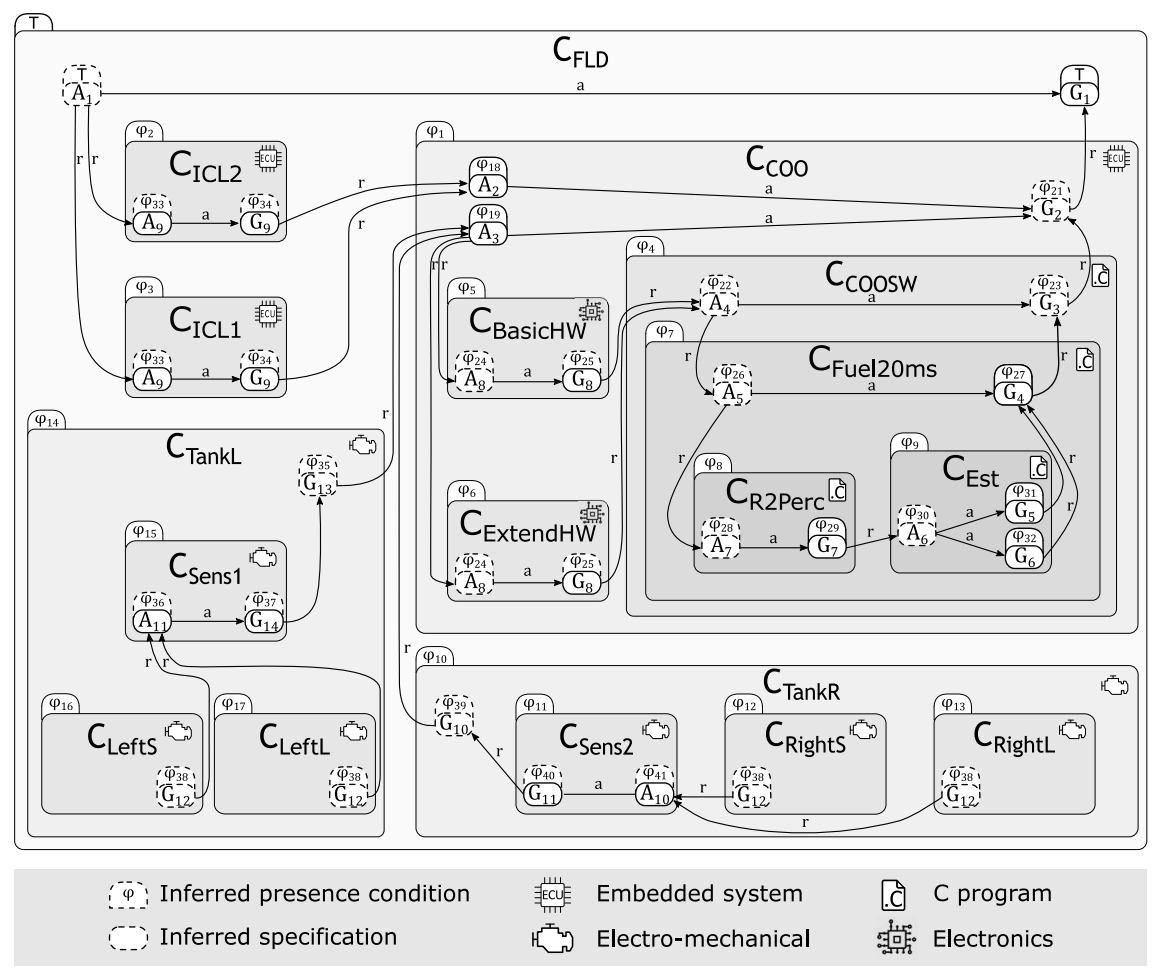

Figure 5.8: CBD model of the FLD system for a considered unit of analysis.

and $A_{11}$. Note that $A_{10}$ and $A_{11}$ abstract away the details of the assumptions to reduce clutter. Finally, because ICL is bought from a supplier, $\mathrm{C}_{\text {ICL1 }}$ and $\mathrm{C}_{\text {ICL2 }}$ were accompanied by a data-sheet that explicitly declared assumptions.

For all the considered components, and all requirements within the documents, except for the requirements within the data-sheets of components bought from suppliers, the presence conditions were explicitly defined. In the analyzed artifacts they were called configuration parameters, and they were actually written according to the grammar from Def. 3, but with different symbols, e.g. instead of symbol $\wedge$, symbol \&\& was used.

As can be seen from Figure 5.8, the explicit requirements within the requirements documents were insufficient to identify the contracts for all considered components. Moreover, there were no traceability links between the 
Table 5.2: Assumptions and guarantees from contracts in Figure 5.8. Underlined A's and G's were declared in the analyzed documents.

\begin{tabular}{|c|c|}
\hline$\underline{\mathrm{G}_{1}} \equiv \mathrm{G}_{2} \equiv \mathrm{G}_{3}$ & $\begin{array}{l}\text { The indicated fuel level shall not deviate more than } \pm 5 \% \text { from the actual } \\
\text { fuel volume AND The indicated fuel level is indicated immediately when } \\
\text { the vehicle is switched on AND In the case of a fault mode, the indicated } \\
\text { fuel level shall be equal to zero. }\end{array}$ \\
\hline$\underline{\mathrm{A}_{9}} \equiv \mathrm{A}_{1}$ & The ignition key is in the $O N$ position. \\
\hline$\underline{\mathrm{A}_{3}} \equiv \mathrm{A}_{8}$ & The sensor resistance value correspond to datasheet specification. \\
\hline$\underline{\mathrm{G}_{9}} \equiv \underline{\mathrm{A}_{2}}$ & $\begin{array}{l}\text { The indicated fuel level shall be displayed in the ICL as a bar-graph, } \\
\text { and controlled by estimated fuel volume [0\%-100\%]. }\end{array}$ \\
\hline$\underline{\mathrm{G}_{4}}$ & $\begin{array}{l}\text { The estimated fuel volume corresponds to the measured fuel vol- } \\
\text { ume AND The estimated fuel level shall be transmitted over CAN every } \\
\text { 2s from when the } C_{C O O} \text { starts AND When the fuel level sensor has } \\
\text { status error or not available, then the estimated fuel level shall be zero. }\end{array}$ \\
\hline$\underline{\mathrm{G}_{5}}$ & $\begin{array}{l}\text { The estimated fuel level corresponds to the measured fuel volume ac- } \\
\text { cording to Table X, or estimated fuel level value equals zero. }\end{array}$ \\
\hline$\underline{\mathrm{G}_{6}}$ & $\begin{array}{l}\text { The estimated fuel level corresponds to the measured fuel volume ac- } \\
\text { cording to Table Y, or estimated fuel level value equals zero. }\end{array}$ \\
\hline $\mathrm{G}_{7} \equiv \mathrm{A}_{6}$ & The percent fuel level shall range from $0 \%$ to $100 \%$. \\
\hline $\mathrm{G}_{8} \equiv \mathrm{A}_{4} \equiv \mathrm{A}_{5} \equiv \mathrm{A}_{7}$ & $\begin{array}{l}\text { The measured fuel level corresponds to the current sensor resistance } \\
\text { according to the formula } V=\left(R_{\text {sens }} \div\left(R_{\text {sens }}+10 K \Omega\right)\right) * 5 \mathrm{~V} \text {. }\end{array}$ \\
\hline $\mathrm{G}_{14} \equiv \mathrm{G}_{13}$ & Sensor resistance ranges linearly from $10 \Omega$ to $180 \Omega$. \\
\hline$\underline{\mathrm{G}_{11}} \equiv \mathrm{G}_{10}$ & $\begin{array}{l}\text { Sensor resistance ranges from } 10 \Omega \text { to } 182 \Omega \text { in } 26 \text { equidistant, discrete } \\
\text { steps. }\end{array}$ \\
\hline$\underline{\mathrm{A}_{10}} \equiv \underline{\mathrm{A}_{11}} \equiv \mathrm{G}_{12}$ & $\begin{array}{l}\text { The electro-mechanical properties of the fuel tank are according to the } \\
\text { fuel-sensor datasheet. }\end{array}$ \\
\hline
\end{tabular}

requirements of different documents, which would correspond to the intended refinement captured by $e^{\mathrm{r}}$ edges in a PL specification structure. However, this was expected because the FLD system was not developed according to CBD principles. Fortunately, this information was possible to infer by triangulating between the available artifacts, as described by the following subsection.

\section{Inferring Missing Information}

Because the ideal outcome of the exploratory case study is to create a proper PL specification structure that can be used for the creation of an assurance 
case, and because our long-term vision is that this process can be automated, the missing information was inferred by triangulating between the available artifacts. Therefore, it was important to avoid introducing any new information. This means that the inference steps are conservative, and that they build a best guess CBD model, which can then be refined if needed.

Missing Guarantees. Regardless of the actual content of a specification that is declared to be a guarantee, there are two conditions of the CBD framework that guarantees must satisfy. Firstly, if the guarantee $G$ belongs to a contract that is allocated to a composite component $C$, then a CBD model must contain an edge $e^{\mathrm{r}}=\left(G^{\prime}, G\right)$ where $G^{\prime}$ is a guarantee of a contract allocated to a subcomponent of $C$. (c.f. Def. 9 (ii)]). Secondly, such $e^{\mathrm{r}}$ edge must correspond to specification refinement (c.f. Corollary 1 (iii)p), e.g. to logical entailment. These two conditions jointly ensure that the specified behavior of a composite component is a consequence of the specified behavior of its subcomponents.

Guided by these two conditions, first, for each component that did not have a contract with a guarantee allocated, a guarantee was declared, i.e. guarantees $\mathrm{G}_{2}, \mathrm{G}_{3}, \mathrm{G}_{10}, \mathrm{G}_{12}, \mathrm{G}_{13}$.

Secondly, to satisfy condition Def. g(i), $e^{\mathrm{r}}$ edges were declared between guarantees $\mathrm{G}_{1}$ through $\mathrm{G}_{6}$, between $\mathrm{G}_{14}$ and $\mathrm{G}_{13}$, and between $\mathrm{G}_{11}$ and $\mathrm{G}_{10}$. For the guarantees $G_{1}$ through $G_{6}$, regardless of their content, there was no alternative way to declare the $e^{\mathrm{r}}$ edges. For the edges $e^{\mathrm{r}}=\left(\mathrm{G}_{14}, \mathrm{G}_{13}\right)$ and $e^{\mathrm{r}}=\left(\mathrm{G}_{11}, \mathrm{G}_{10}\right)$, an alternative was to state that guarantee $G_{12}$ should refine $G_{13}$ and $G_{10}$. However, this would mean that $\mathrm{C}_{\mathrm{Sen} 1}$ and $\mathrm{C}_{\mathrm{Sens} 2}$ do not contribute to the behavior of $\mathrm{C}_{\text {TankL }}$ and $\mathrm{C}_{\text {TankR }}$, and consequently the explicit assumption $\mathrm{A}_{3}$ would not be refined by any specification.

Thirdly, the actual content of the guarantees was inferred, such that condition Corollary 1 (iii)) was satisfied. The special case in which refinement trivially holds, regardless of how refinement is defined, is when two specifications are equivalent. This principle was adopted to avoid adding any new information, i.e. the content of the inferred guarantees was declared equivalent to the existing requirements from the analyzed requirements documents. For example, $\mathrm{G}_{2} \equiv \mathrm{G}_{1}$ practically means that the functionality of the FLD system, i.e. the fact that the estimated fuel volume corresponds to the actual fuel volume, is a consequence of the functionality of the COO ECU, whose software computes the estimated fuel volume.

Missing assumptions. Any assumption within a CBD model must satisfy two conditions. Firstly, except the overall system assumption, for each assump- 
tion $A_{i}$ of a contract allocated to component $C$, the CBD model must contain an edge $e^{\mathrm{r}}=\left(S, A_{i}\right)$ where $S$ is either an assumption of a parent of $C$, or a guarantee of a sibling of $C$ (c.f. Def. 9 (i) ) ). Secondly, this edge must correspond to specification refinement (c.f. Corollary 1 1iii)).

Guided by these conditions, firstly an assumption was declared for each contract without an assumption. Exceptions are electro-mechanical components $\mathrm{C}_{\text {TankL }}, \mathrm{C}_{\text {TankR }}, \mathrm{C}_{\text {LeftS }}, \mathrm{C}_{\text {LeftL }}, \mathrm{C}_{\text {Rights }}, \mathrm{C}_{\text {RightL }}$ because their assumptions would correspond to acceptable types of fuels, or allowed fuel pressure, and these were deemed out of scope. Unlike for missing guarantees, the $e^{\mathrm{r}}$ edges and the content of newly declared assumptions were inferred simultaneously.

Assumption $A_{2}, A_{3}, A_{6}, A_{10}$, and $A_{11}$ are the assumption intended to be refined by guarantees. Regarding $A_{2}$, it was explicitly equivalent to $\mathrm{G}_{9}$ and an $e^{\mathrm{r}}$ edge was asserted. This is a great example of contract thinking in industry where one company assumes a component that satisfies $\mathrm{A}_{2}$, and then outsources the development of such component but from the point of view of the supplier this is a guarantee, namely $\mathrm{G}_{9}$. Regarding $\mathrm{A}_{3}$, the content of $\mathrm{A}_{3}$ clearly could only be refined by a guarantee of a sensor, and because the guarantees of sensors were equivalent to guarantees of $\mathrm{C}_{\text {TankL }}$ and $\mathrm{C}_{\text {TankR }}$, the corresponding $e^{\mathrm{r}}$ edges were asserted. Regarding $A_{6}$, the inspection of the source code revealed that the input of $\mathrm{C}$-function $\mathrm{C}_{\mathrm{Est}}$ was the output of function $\mathrm{C}_{\mathrm{R} 2 \mathrm{Perc}}$. Therefore, the assumption $A_{6}$ was concluded to be equivalent to guarantee $G_{7}$ and the edge $e^{\mathrm{r}}=\left(\mathrm{G}_{7}, \mathrm{~A}_{6}\right)$ was added, in accordance with Def. 99 (i)). Regarding $\mathrm{A}_{10}, \mathrm{~A}_{11}$, and corresponding $e^{r}$ edges, the data-sheet of the two sensors explicitly stated that the corresponding fuel tanks must refine their assumptions.

Regarding the remaining assumptions, they have an incoming $e^{\mathrm{r}}$ edge from another assumption, according to Def. 95 (i). Regarding $A_{7}$, the corresponding guarantee $G_{7}$ guarantees the value percent fuel level. By inspecting the source code, component $\mathrm{C}_{\mathrm{R} 2 \mathrm{Perc}}$ takes as input the measured fuel value from the analogue-to-digital converter which is a part of components $\mathrm{C}_{\mathrm{BasicHW}}$ and $\mathrm{C}_{\text {ExtendHw. }}$. Consequently, there should exist an $e^{\mathrm{r}}$ edge from $\mathrm{G}_{8}$ to $\mathrm{A}_{7}$, but such edge is not allowed according to Def. 9 (i) ). Instead, the allowed edges are from $A_{5}$ to $A_{7}$ and from $A_{4}$ to $A_{5}$. Then, because $A_{4}$ belongs to a contract that is allocated to a sibling component of $\mathrm{C}_{\text {BasicHW }}$ and $\mathrm{C}_{\text {ExtendHW }}$, the edge $e^{\mathrm{r}}=\left(G_{8}, A_{4}\right)$ can be asserted and also the content of these guarantees is defined as $\mathrm{G}_{8} \equiv \mathrm{A}_{4} \equiv \mathrm{A}_{5} \equiv \mathrm{A}_{7}$. Similar reasoning was applied to infer the content and the edges involving $A_{8}$. Finally, assumption $A_{1}$ was inferred because the explicit assumption $A_{9}$ refers to the ignition key of the vehicle, and because the ignition key is not a component of the FLD system, then this assumption can only be 
Table 5.3: A subset of the presence conditions from Figure 5.8. Underlined $\varphi^{\prime} s$ were declared in the analyzed artifacts.

\begin{tabular}{|c|c|}
\hline$\underline{\varphi_{1}}$ & $\begin{array}{l}\left(f_{2482-1} \vee\left(f_{2482-2} \wedge f_{1940-2}\right) \vee\left(f_{2482-2} \wedge f_{1940-1}\right)\right) \wedge\left(f_{1837-1} \vee\right. \\
\left.\left(f_{1837-19} \wedge f_{579-26}\right)\right)\end{array}$ \\
\hline$\underline{\underline{\varphi_{4}}}$ & $\varphi_{1} \wedge f_{1323-5}$ \\
\hline$\varphi_{7}=\varphi_{9}$ & $\left(f_{1-1} \vee f_{1-2}\right) \wedge \neg f_{520-2}$ \\
\hline$\underline{\underline{\varphi_{5}}}$ & $\begin{array}{l}\left(f_{2482-1} \vee\left(f_{2482-2} \wedge f_{1940-1}\right) \vee\left(f_{2482-2} \wedge f_{1940-1}\right)\right) \wedge\left(f_{1837-1} \vee\right. \\
\left.\left(f_{1837-19} \wedge f_{579-26}\right)\right) \wedge\left(\left(f_{37-1} \wedge f_{2519-1}\right) \vee\left(f_{37-3} \wedge f_{2519-1}\right) \vee\right. \\
\left.f_{37-5}\right)\end{array}$ \\
\hline$\underline{\underline{\varphi_{6}}}$ & $\begin{array}{l}\left(f_{2482-1} \vee\left(f_{2482-2} \wedge f_{1940-1}\right) \vee\left(f_{2482-2} \wedge f_{1940-1}\right)\right) \wedge\left(f_{1837-1} \vee\right. \\
\left.\left(f_{1837-19} \wedge f_{579-26}\right)\right) \wedge\left(\left(f_{37-2} \wedge f_{2519-1}\right) \vee\left(f_{37-4} \wedge f_{2519-1}\right) \vee\right. \\
\left.f_{37-5}\right)\end{array}$ \\
\hline$\varphi_{24}=\varphi_{25}$ & $\varphi_{5} \vee \varphi_{6}$ \\
\hline$\underline{\varphi_{23}}=\underline{\varphi_{27}}$ & $\left(f_{1-1} \vee f_{1-2}\right) \wedge f_{520-1}$ \\
\hline$\underline{\underline{\varphi_{31}}}$ & $f_{3431-26} \wedge f_{3706-29} \wedge f_{4323-26}$ \\
\hline$\underline{\varphi_{32}}$ & $f_{3431-26} \wedge f_{3706-28} \wedge f_{4323-26}$ \\
\hline
\end{tabular}

refined by a specification allocated to a component outside the FLD system. In other words, assumption $\mathrm{A}_{9}$ must be an assumption of the overall FLD system.

Missing presence conditions. Besides the missing presence conditions of specifications of supplier-bought components, the presence conditions of inferred assumptions and guarantees had to be inferred. Table 5.3 shows examples of identified and inferred presence conditions. The full list is omitted because some expressions are very large, e.g. the expression for $\varphi_{15}$ contains over 45 terms.

Missing presence conditions of specifications that are allocated to electronic and electro-mechanical components were inferred to be equivalent to the presence conditions of these components because the behavior of such components is unchangeable. When the same specification was allocated to several electronic or electro-mechanical components, the presence conditions was declared to be a disjunction of the presence conditions of different components. In this way, presence conditions $\varphi_{5}, \varphi_{24}, \varphi_{25}$, and $\varphi_{33}-\varphi_{41}$ were inferred. For example, as Table 5.3 shows, presence conditions $\varphi_{24}$ and $\varphi_{25}$ were set to $\varphi_{5} \vee \varphi_{6}$. The remaining presence conditions, namely $\varphi_{22}, \varphi_{23}$, and $\varphi_{26}-\varphi_{30}$ were inferred to 
satisfy conditions of Theorem 3 i.e. to ensure that if a specification $S_{1}$ is intended to be refined by another specification $S_{2}$, then the presence condition of $S_{2}$ should entail the presence condition of $S_{1}$. To avoid adding new information, in the same way as for assumptions and guarantees, this was done by asserting that $p\left(S_{1}\right)=p\left(S_{2}\right)$. For example, as Table 5.2 shows, $\varphi_{23}$ was inferred to be equivalent to $\varphi_{27}$.

\subsection{Results}

This section answers the formulated questions. The answer to each question is contextualized with the lessons learned during the synthesis of the CBD model.

\section{Answering Q1}

When it comes to question Q1, the answer is that in general it was possible to represent a real, industrial product-line as a CBD model, but several inference steps had to be performed. On the one hand, this was expected, because the presented CBD framework was not used when the FLD system was developed. The inference steps were also expected because the CBD model represents the complete FLD system, from the most abstract components to the detailed software components, and such representation of the FLD system did not exist. To a good extent, the inferences were needed because of the practices in SCANIA, namely because requirements were written for logical components, which are then interpreted by developers to create the technical components which implement the logical components. Using the terminology of the domain safety-standard ISO 26262 , the practice was to explicitly declare functional safety requirements, but not the technical and software safety requirements. Given that since 2018, when a version of ISO 26262 applicable to trucks was released, the FLD system was brought closer to the guidelines of ISO 26262. Thus, applying the CBD framework to the newest version would require less inference effort.

On a positive note, guided by the conditions of the CBD framework, the necessary inferences were possible to perform only by triangulating between the available artifacts. Out of tens of different conditions, it was sufficient to consider only five of them. These are the two conditions that define the allowed relations between assumptions and guarantees (c.f. Def. 99.i) and Def. 9(ii) ), the two conditions that define the required entailment between presence conditions (c.f. Theorem 3), and condition (iii) of Corollary 1. Also note that given our long term vision to create CBD model in an automated fashion, the CBD model was synthesized without any manual effort by domain experts. Although 
this means that the synthesized CBD model is a conservative, best-guess model, it is important to note that even if a system, such as the FLD system, is deployed and currently in the maintenance phase, it is possible to create an initial CBD model by using the presented inference steps. Then, the obtained CBD model can be used a starting point for further refinement by domain experts.

\section{Answering Q2}

Regarding question Q2, first it must be considered if the model in Figure 5.8 satisfies all conditions of the CBD framework. The synthesized CBD model is a proper PL specification structure, according to Def. 12, as required by Corollary 1. The model also satisfies condition (iii) of Theorem 3, i.e. the model does not contain directed cycles. Furthermore, the FLD system satisfies condition (iv) of Corollary 1 because for each atomic component, there was a positive verification result in the verification documents. Note that because the FLD system was developed in a non-compositional way, additional verification was performed for integration of SW and HW component into ECUs, and for the integrated FLD system.

However, when it comes to the remaining conditions of Corollary 1, the situation is less favorable. The model in Figure 5.8 does not satisfy condition (iii) of Corollary 1, i.e. it is not the case that each $e^{r}$ edge corresponds to specification refinement. For example, even without a formal analysis it is clear that $\mathrm{G}_{4}$ does not refine $G_{3}$ because $G_{3}$ guarantees that a value of indicated fuel level is available immediately when the vehicle is switched on, but $\mathrm{G}_{4}$ produces a value of estimated fuel level that can be outputted to $\mathrm{C}_{\text {ICL1 }}$ or $\mathrm{C}_{\text {ICL2 } 2}$ every $2 \mathrm{~s}$. Similar problems exist between $G_{13}$ or $G_{10}$ and $A_{3}$. Given the fact that the verification documents showed positive testing results, and the fact the FLD system has been deployed for years, the most probable explanation is that during the implementation these inconsistencies among the specifications were identified and corrected, but the modifications were not propagated back to the requirements. If the CBD framework had been used at the time of development, these inconsistencies would have been identified before the implementation phase. Detecting inconsistent and incomplete system design early is particularly important for highly critical systems whose development is expensive.

Regarding the remaining conditions of Theorem 2, and Theorem 3 , it is unclear if they are satisfied. Because these conditions include the corresponding variability model, and because SCANIA variability model contains around 50.000 features, it was outside the scope of the present paper to actually verify these conditions. However, although explicitly searched for, no verification artifact 
was identified that performs this kind of analysis. Therefore, a clear action for SCANIA is to start performing such analysis to ensure the absence of configuration mismatches. Currently, in line with common industrial practice [62, the absence of configuration mismatches is partially verified during testing, because the identified verification documents contained testing results for several frequently ordered FLD variants. However, given that the total number of variants is around 24.000, the variant coverage is rather low.

In summary, given the analyzed artifacts of the FLD system, and the conditions of Corollary 1, it cannot be said that the FLD system satisfies the contract $\left(\left\{A_{1}\right\}, G_{1}\right)$ in each possible configuration of a SCANIA vehicle. However, the identified inconsistencies between the specification and the implementation point to possible improvements of the FLD system-design. Moreover, the lack of exhaustive analysis for the absence of configuration mismatches, which is precisely formalized in Theorem 2 and Theorem 3, can be translated to an additional step in the engineering process that would increase the quality of SCANIA system. Therefore, as an answer to Q2, the use of the CBD framework can indeed lead to actionable engineering insights to improve the engineering process.

To set the answer to Q2 into a realistic context with respect to the required effort, note that using the CBD framework implies the need for a higher number of artifacts and traceability links, of higher quality. Compared to the practices observed in the case study this would certainly require the introduction of new processes and methods in the engineering process. Alternatively, construction of the CBD model may be performed on-demand from legacy artifacts, as shown in Section 6.3. The benefit of accepting the overhead may be seen from the observed mismatch between the FLD requirements and the corresponding implementation. This mismatch shows that not using a rigorous framework in design stages, must be compensated in implementation stages, but with a much higher effort. Detecting inconsistencies between the requirements in implementation stages means that implementation has been done with respect to inconsistent requirements, then verification has revealed faults, which lead to changes to the implementation, which in turn must be verified again.

If the alternative is chosen, and CBD models are created on-demand from legacy artifacts, this implies a lesser but not an insignificant effort. In the case of the FLD system, the biggest effort was spent on the manual identification and collection of the relevant artifacts from different engineering tools and databases. This activity required about one person-week, but it should be noted that the relevant artifacts were in data-silos and in a more-digitalized toolchain this activity would be shorter. Given the relevant artifacts, the CBD model was effectively created in two person-days. Because the unit of analysis 
included around one third of the total FLD system, creating the CBD model for the complete system would require additional effort. On the other hand, because the FLD system was already deployed, the reported effort corresponds to the creation of the model at the end of the development process. Since the benefits of the CBD framework materialize if used during the development, a more realistic scenario is that the CBD model is created iteratively, in parallel with the development process, thus reducing the overall effort.

\section{An Assurance Case for a Product Line}

This section presents the second contribution, which is a method to construct assurance-case arguments, based on a CBD model of a product line. The method allows the construction of product-based arguments [40] that argue that a system satisfies a particular property in all configurations. Moreover, the method produces a modular argument in order to facilitate assurance-case maintenance, and to facilitate the creation of smaller pieces of argumentation in isolation, and their subsequent integration.

Because the CBD framework is notation independent, the following method is general. In this context, being general means that the method can be used to represent and reason about different dependability properties, e.g. safety, reliability, or security, for configurable systems of different size, and from different domains. This section relies on the GSN format to express assurance cases, but note that the GSN syntax has been mapped to Structured-Assurance Case Metamodel (SACM) [36, 80, which is an OMG standard that defines the abstract syntax for different concrete syntaxes that can be used to express an assurance case. Before defining the assurance-case construction method, the elements of the GSN syntax, which are relevant for the present paper, are introduced.

\subsection{Goal-Structuring Notation}

Besides the Claims-Arguments-Evidence (CAE) notation [2], NOR-STA notation [34, and the concrete syntax for SACM [36], the GSN notation 68] is the most prominent graphical syntax for expressing assurance cases. GSN is "a graphical argument-notation which can be used to document explicitly the elements, and the structure of an argument and the argument's relationship to evidence" [68]. More specifically, GSN models form rooted graphs called goal-structures, which capture the assurance-case argumentation structure. Figure 5.9 shows the GSN elements used in the present paper. A complete de- 
scription of the GSN notation can be found in [68, and formalization of the goal-structures can be found in [21].

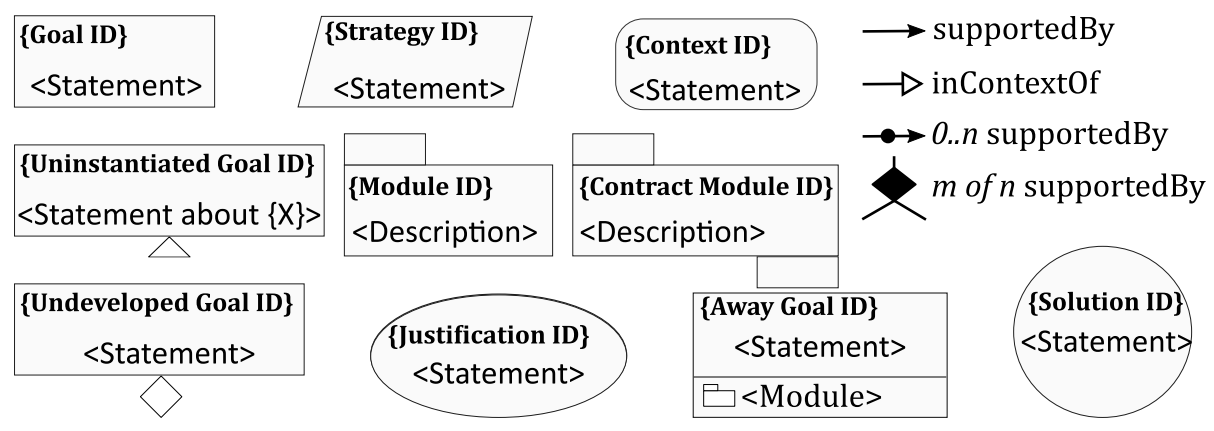

Figure 5.9: GSN elements used in the present paper.

The elements in Figure 5.9 have the following meaning: (i) Goal represents a claim about the system, (ii) Strategy represents the rationale for decomposing a goal into subgoals, (iii) Solution represents the evidence expected to support a claim within a goal, (iv) Justification explains why the rationale within a strategy is sound, (v) Context declares the context in which the claim should be interpreted, (vi) Module is a container to encapsulate smaller, self-contained goal-structures in order to facilitate the management of large assurance cases, (vii) Away Goal is a reference to a goal that is defined within a particular Module, and (vii) a Contract Module is a special type of module that defines how a goal within one module is supported by a goal from another module. Note that GSN contract modules are not directly related to the CBD design contract. The purpose of GSN contract modules is to decouple the argumentation between dependent GSN modules, while the purpose of CBD contracts is to modularly specify the behavior of a system.

The described elements can be connected through the supportedBy and inContextOf links. The connections between the elements from Figure 5.9 obey the following rules:

- permitted supportedBy connections are goal-to-goal, goal-to-strategy, goalto-solution, strategy-to-goal, goal-to-away goal, goal-to-module, goal-to-contract module, strategy-to-away goal, strategy-to-module, and finally strategy-tocontract module

- permitted inContextOf connections are goal-to-context, goal-to-justification, 
strategy-to-context, strategy-to-justification, goal-to-away goal, goal-to-module, strategy-to-away goal, and finally strategy-to-module.

To allow specifying template goal-structures, i.e. assurance case patterns [54, additional GSN elements have been defined with the following meaning: (i) Uninstantiated Goal represents a claim that contains parameters which must be instantiated for a particular system, e.g. parameter X in Figure 5.9 (ii) Undeveloped Goal represents a claim that must be further developed, i.e. connected to further goals, (iii) the supportedBy link with a black circle defines that the multiplicity of the supportedBy link is zero to many, and (iv) a black diamond splitting a supportedBy link into several ones is the so called choice element and it defines that $m$ out of $n$ supportedBy links are necessary for the argument to be complete.

\subsection{Overview of the CBD-Based Pattern}

This section presents the main idea for the assurance-case argumentation-creation method, namely a modular assurance-case pattern, which is based on the conditions of the CBD framework. More precisely, the method is based on two, mutually dependent patterns, which are shown in Figure 5.10 and Figure 5.10. The idea is that for each component $C$, the pattern in Figure 5.10 will yield the argumentation that $C$ satisfies the allocated contracts in all configurations. This captures the idea that each component can be developed in isolation with respect to its contract, i.e. with respect to the acceptable environments as defined by the declared assumptions (c.f. Def. 6). In addition, for all non-root components in the PL architecture, the pattern in Figure 5.12 will yield the argumentation that within a particular system, i.e. represented by the root component, the assumptions of contracts are satisfied by other components comprising the system. In other words, once the different components are integrated into a system, the environment of each non-root component is fixed and it must be shown that this environment is indeed acceptable, i.e. it satisfies the declared assumptions. The reason why the pattern in Figure 5.12 does not apply to the root component, i.e. to the overall system, is because the assumptions of the system are effectively assumptions on the intended deployment environment of the system. For example, $\mathrm{A}_{1}$ in Table 5.2 places an assumption on the driver of the vehicle.

Another important point is that the patterns induce a modular architecture in order to enable creation of smaller, self-contained arguments that capture conceptually different arguments. This means that for each component that 
instantiates the patterns from Figure 5.10 and Figure 5.10, the corresponding modules with the argumentation from the two patterns will be created. In this way, the overall argument is more robust with respect to possible assurancecase changes, but it also supports the allocation of responsibilities for particular modules to different engineering groups.

\section{Description of the Patterns}

To distinguish between the elements of the patterns, we adopt the prefixes PC (Pattern for Component) and PI (Pattern for Integration) for element identifiers. Also note that some goals are both uninstantiated, and undeveloped. Here undeveloped means that once instantiated, these goals should be supported by evidence.

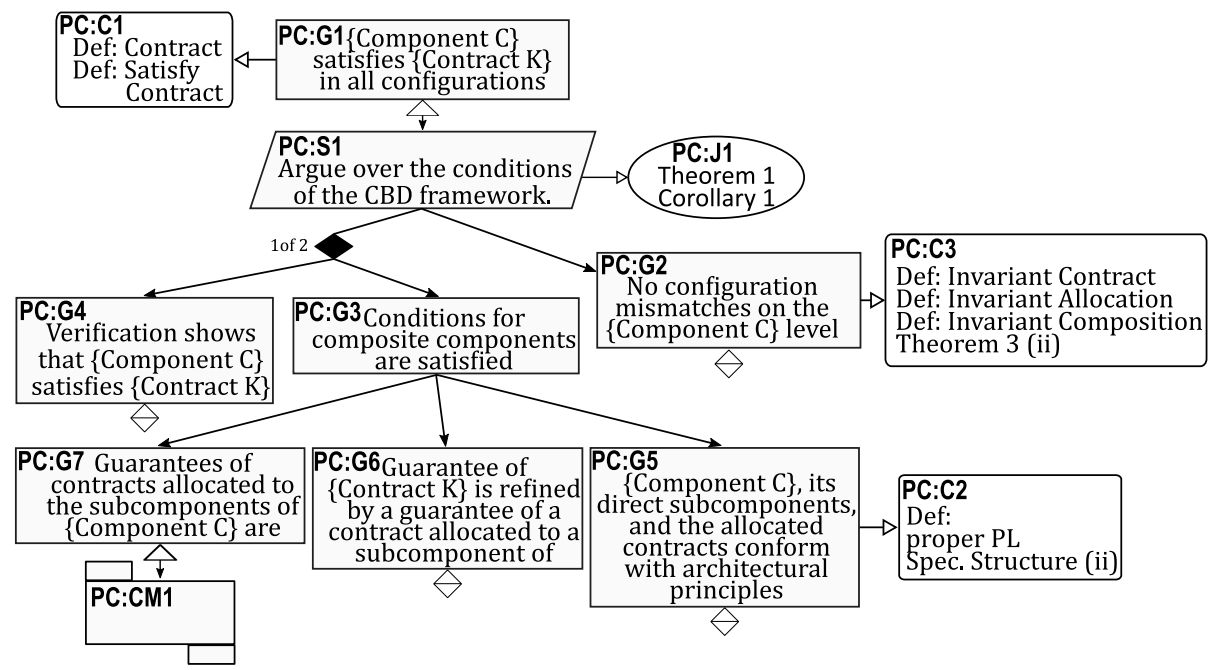

Figure 5.10: Environment-independent pattern for each component C

As indicated by goal PC:G1, the goal structure in Figure 5.10 applies to any component $\mathrm{C}$ that has a contract $\mathrm{K}$ allocated to it. Recall that a component can represent any type of component, at any abstraction level, e.g. a complete product such as a vehicle, or a single software function. Strategy PC:S1 states that the claim in PC:G1 holds if the system represented by component $\mathrm{C}$ satisfies the conditions of the CBD framework. This strategy is asserted in 
the context of justification PC:J1, which references Theorem 1 and its product line extension in the form of Corollary 1. The remaining goals within the pattern claim that the conditions of the CBD framework are satisfied. Goal PC:G2 claims that there are no configuration mismatches, as defined by the four invariance conditions in PC:C3. Note that Def. 16 and Theorem 3 ii) apply only to composite components as they define invariant composition and absence of mismatches between the guarantee of a composite component and the guarantees of its direct subcomponents.

Goals PC:G3 and PC:G4 are mutually exclusive, as indicated by the choice element between them and the strategy element PC:S1. According to Corollary 1 iv), goal PC:G4 claims that verification shows that component $\mathrm{C}$ satisfies the allocated contract, if $\mathrm{C}$ is an atomic component. Goal PC:G3 groups goals PC:G5-PC:G7, which are instantiated if $\mathrm{C}$ is a composite component. Goal PC:G5 claims that $\mathrm{C}$ and its direct subcomponent conform with the architectural principles, which correspond to the conditions for a proper PL specification structure (c.f. Def. 12). Goal PC:G6 claims that the guarantee of contract K is indeed refined, as required by Corollary 1 iii), by some guarantee of a contract allocated to a subcomponent of $\mathrm{C}$, as defined by Def. 9. Finally, goal PC:G7 claims that the guarantees of contracts allocated to subcomponents of $\mathrm{C}$ are satisfied, i.e. the subcomponents of $\mathrm{C}$ behave as specified. Note that formally, the CBD framework defines the satisfies relation between a component and a specification while PC:G7 does not precisely declare which component satisfies a specific guarantee. However, the idea is that from the perspective of component $\mathrm{C}$, the subcomponents can be unknown, or can change occasionally. Thus, goal PC:G7 is refined with respect to particular components and specifications within the contract module PC:CM1, which supports the goal PC:G7. In other words, contract module PC:CM1 decouples the argumentation that component $\mathrm{C}$ satisfies its contracts, from the argumentation about its possibly unknown subcomponents. As can be seen from Figure 5.11, the contract module binds the module of component $\mathrm{C}$, i.e. the goal PC:G3, with the modules obtained by instantiating the pattern from Figure 5.12 for each subcomponent of C.

When it comes to the pattern in Figure 5.12 , PI:G1 claims that the composition of component $\mathrm{C}$ with its environment, i.e. with other components of the system, satisfies the guarantee of each contract allocated to C. The strategy in PI:S1 states that the argument is over each contract allocated to component C. As discussed previously, for a guarantee of a contract to be satisfied, the assumptions of the contract must be satisfied by the environment, i.e. refined by a specification that is satisfied by another component of the system. This claim is 


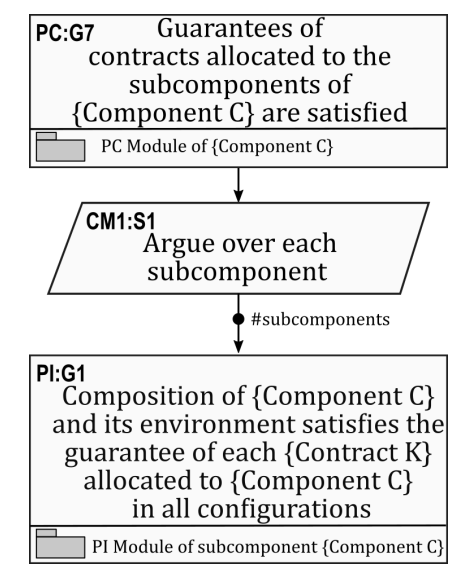

Figure 5.11: Contents of the contract module PC:CM1.

captured by goal PI:G3 and it corresponds to condition Def.9 i) which defines the specifications that can refine an assumption, and to condition Corollary 1 iii) which requires that the refinement holds (c.f. PI:C1). For an assumption to be satisfied in all configurations, the refines relation between each assumption and the specification that refines it, must satisfy condition Theorem 3 i) as captured by PI:G2 and PI:C2. Finally, given an appropriate environment as claimed in PI:G2 and PI:G3, the component $\mathrm{C}$ must satisfy the allocated contract in all configurations. Because this claim is the root claim of the pattern in Figure 5.10, an away goal PC:G1 refers to the root goal of pattern in Figure 5.10. Note that instead of an away goal, a contract module could have been used. However, because the argument about the integration in a particular environment cannot be reused in any other environment, and also because the pattern in Figure 5.10 can be instantiated only once for a particular component and corresponding contracts, decoupling the modules with a contract module would not bring significant benefits.

The fact that the patterns induce a modular architecture brings two benefits. Firstly, monolithic assurance cases are avoided and smaller pieces of argumentation can be created separately within particular models. Secondly, the assurance case modules, and the corresponding argumentation, can be created in parallel with the development process. For example, as the development of a component progresses, the pattern in Figure 5.10 can be gradually instantiated and evidence created. Then, when the integration of the component starts, the pat- 


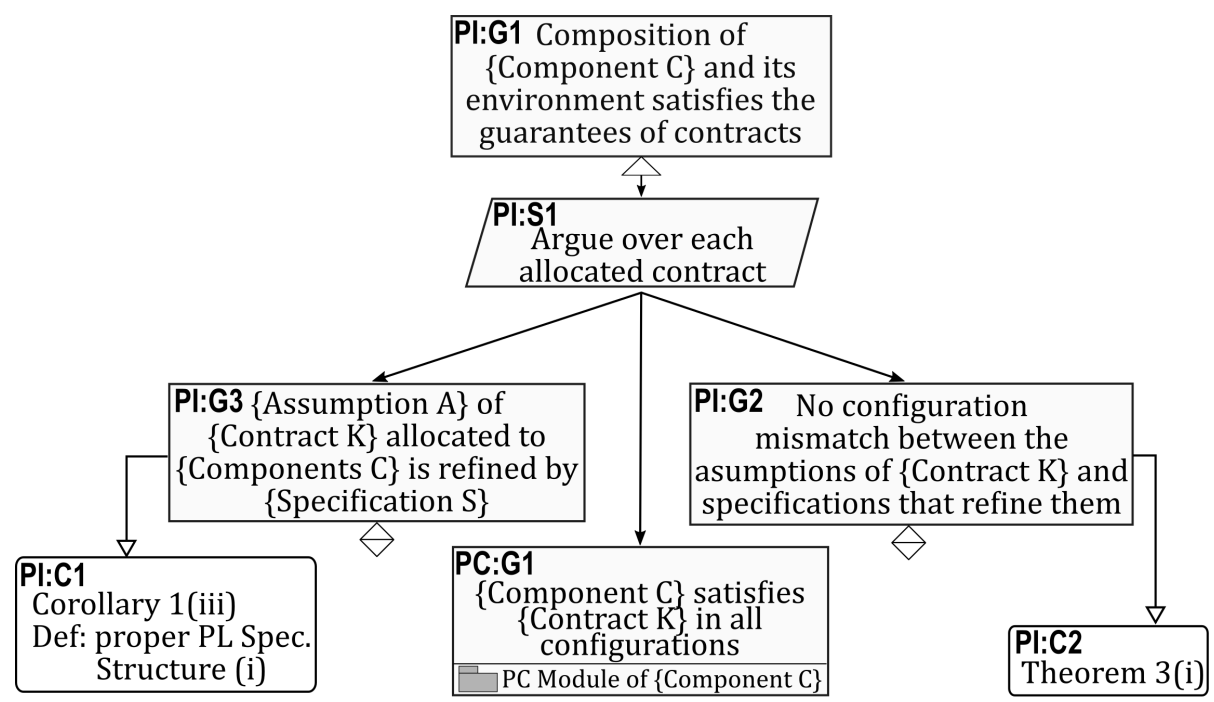

Figure 5.12: Pattern for the integration of component $\mathrm{C}$ with its environment

tern in Figure 5.12 can be gradually instantiated and evidence created. In this way, the effort to create the assurance case is distributed across the development process and any given point in time, the focus is on particular modules of the assurance case, and not on its entirety.

Given the patterns from Figure 5.10 and Figure 5.10 , the following section defines the instantiation procedure, and exemplifies the application of the pattern for the FLD system.

\subsection{Pattern Instantiation}

In this section we define the procedure to instantiate the patterns from Figure 5.10 and Figure 5.10 The procedure is presented as a high-level pseudocode to define the order in which different elements are instantiated, and also to define the correct CBD entities that can instantiate individual pattern elements. The instantiation procedure for the environment independent pattern from Figure 5.10 comprises the following steps:

ei1) Create a module for each component C, instantiate goal PC:G1 for each allocated contract and create a single context element PC:C1, 
ei2) Create a GSN element PC:S1 per instantiated goal PC:G1, and create a single $\mathbf{P C}: \mathbf{J} \mathbf{1}$,

ei3) Instantiate goal PC:G2, and create the context element PC:C3. Def. 16 and Theorem 3 ii) should be a part of PC:C3 only if component $\mathrm{C}$ is composite,

ei4) If component $\mathrm{C}$ is atomic, instantiate goal PC:G4,

ei5) If component $\mathrm{C}$ is composite, instantiate goals PC:G5-PC:G7, and create context PC:C2,

ei6) Create solution elements that support the instantiated goals P:G2, and either P:G4, or P:G5 and P:G6,

ei7) If pattern from Figure 5.12 has been instantiated for subcomponents of component C, instantiate contract module PC:CM1.

The instantiation procedure for the environment dependent pattern from Figure 5.12 comprises the following steps:

ed1) Create a module for each non-root component $\mathrm{C}$ and instantiate goal PI:G1,

ed2) For each assumption of each contract allocated to C, instantiate goal PI:G3 and create context PI:C1,

ed3) For all contracts allocated to component C, instantiate goal PI:G2 and create context PI:C2,

ed4) Create solution elements that support the instantiated goals PI:G3 and PI:G2,

ed5) If the pattern from Figure 5.10 has been instantiated for component C, instantiate the away goal PC:G1.

In conjunction with Figure 5.10 and Figure 5.10 , steps s1-s7 and s1-s5 should be possible to convert into an algorithm that can be used to automate the creation of the assurance-case arguments. Such automation of assurance-case creation would go hand-in-hand with the long term vision to automate the creation and analysis of CBD models.

To exemplify the use of the instantiation procedure, Figure 5.13 shows a part of the modular architecture for the assurance case for the FLD system. 


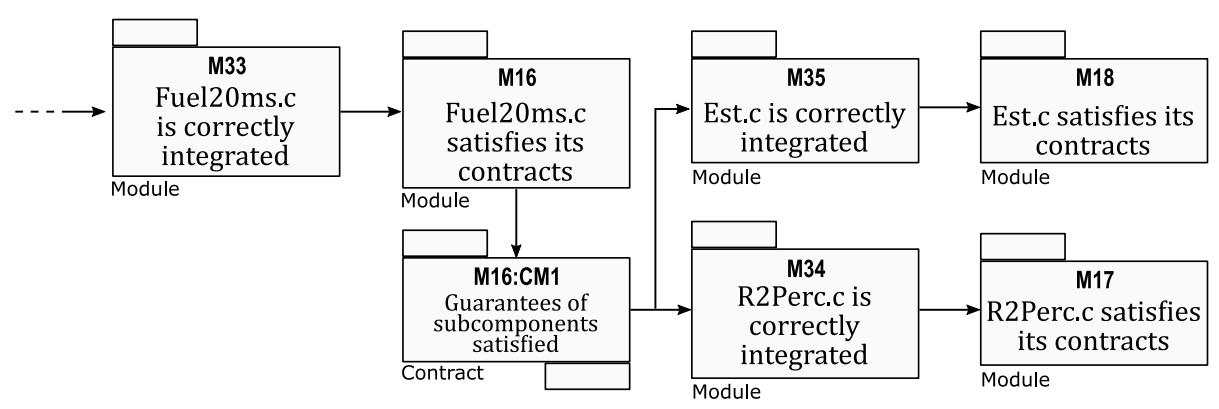

Figure 5.13: Module view for a part of the assurance case for the FLD system.

The complete modular architecture can be found in [1]. Figure 5.13 shows two modules for the SW components Fuel20ms.c, Est.c, and R2Perc.c, where the modules contain the argumentation obtained by instantiating the patterns from Figure 5.10 and Figure 5.10. According to the pattern in Figure 5.10, module M16 contains the argumentation that Fuel20ms.c satisfies the allocated contract since, besides the claims in M16, the subcomponents of Fuel20ms.c, namely R2Perc.c and Est.c, are integrated into their environment so that they satisfy the guarantees of their contracts. This argumentation is contained in modules M34 and M35, where the contract module M16:CM1 relates M16 to M34 and M35. According to the pattern in Figure 5.12, besides the claims within M34 and M35, these modules claim that R2Perc.c and Est.c satisfy the allocated contracts, but through away goals that belong to modules M17 and M18.

To illustrate the content of the different modules, Figure 5.14 shows the content of module M16, the content of module M35 is shown in Figure 5.15, Figure 5.16 shows the content of module M18, Figure 5.17 shows the content of the contract module M16:CM1, and the content of the remaining modules can be found in [1].

The content of module M16 in Figure 5.14 is a straightforward instantiation of the uninstantiated goals with the component Fuel20ms.c The content of module M35 is more involved because the CBD model of the FLD system must be carefully considered. Goal M35:G3 claims that, according to Def. 9 i) and Corollary 1 iii), $A_{6}$ is refined by $G_{7}$. An inspection of the CBD model in Figure 5.8 shows that there exists an edge $e^{r}=\left(\mathrm{G}_{7}, \mathrm{~A}_{6}\right)$ which declares the intention that $G_{7}$ should refine $A_{6}$. Goal M35:G2 claims that according to Theorem 3 i), there is no configuration mismatch between $G_{7}$ and $A_{6}$. More 


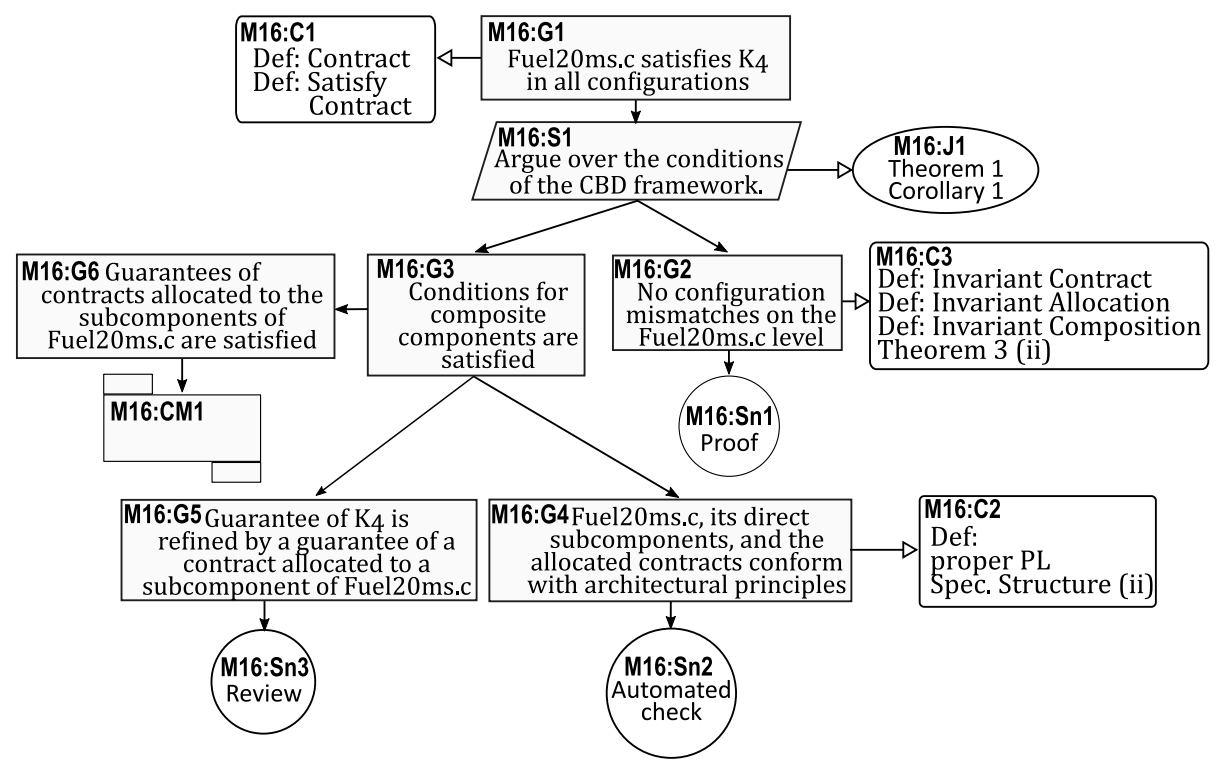

Figure 5.14: Content of module M16.

precisely, it should hold that $p\left(\mathrm{G}_{7}\right) \models_{\Theta} p\left(\mathrm{~A}_{6}\right)$. The claims in M35:G2 and M35:G3 ensure that the environment of Est.c satisfies the assumptions of the contract allocated to Est. $\mathrm{c}$ in all configuration. For the goal M35:G1 to hold, it is also necessary to show that Est.c satisfies the allocated contracts, namely $\left(\left\{A_{6}\right\}, G_{5}\right)$ and $\left(\left\{A_{6}\right\}, G_{6}\right)$. These claims are captured by the away goals M18:G1 and M18:G2.

Because module M18 contains the argumentation that component Est.c satisfies two contracts, and because these two contracts share the assumption $\mathrm{A}_{6}$, the argumentation that the two contracts are satisfied by Est.c share goal M18:G4, context nodes M18:C1 and M18:C2, and the justification node M18:J1. Besides these elements, and because Est.c is an atomic component, the module claims that verification shows that the two contracts are satisfied. In more general terms, this means that a single module contains the argumentation that a component satisfies each allocated contract. Note that because Est.c is an atomic component, module M18 does not refer to further modules, i.e. M18 is the leaf module in the modular architecture of the assurance case for the FLD system. 


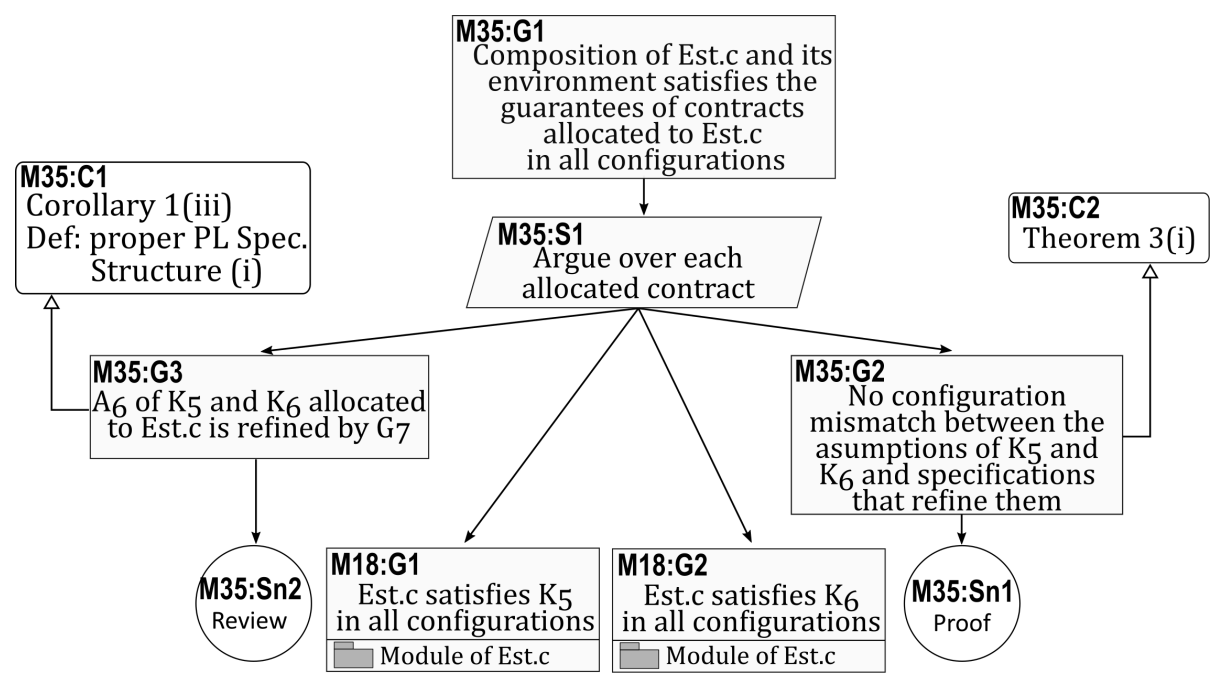

Figure 5.15: Content of module M35.

\subsection{Relating CBD-Based Claims to Other Types of Claims}

As mentioned previously, the argumentation obtained by using the patterns from Figure 5.10 and Figure 5.10 must be integrated with other types of claims to obtain a complete assurance case. An advantage of CBD-based argumentation is that it inherently supports connections to several other types of claims.

Firstly, and as noted in [47, certifying a product line must include the argumentation that the mechanisms for product line management are effective and reliable. More precisely, this means that it should be possible to determine with certainty whether all configurations that can be selected from a variability model are valid, whether a particular artifact applies to the intended configurations, and whether each variant (c.f. Def. 13) derived from the product line is valid. All these types of claims can be developed from the CBD framework.

Throughout the present paper, it was assumed that only valid configurations can be selected from a variability model $\mathfrak{M}$ (c.f. Def. 1). Automated analysis of variability models has been investigated for decades [6], where the analysis of whether a selected configuration is valid, is one of the most basic analysis. Moreover, it has also been shown that automated analysis of very large feature models is a tractable task [59]. Another assumption throughout the present paper is that each presence condition $\varphi$ is consistent with the variability model 


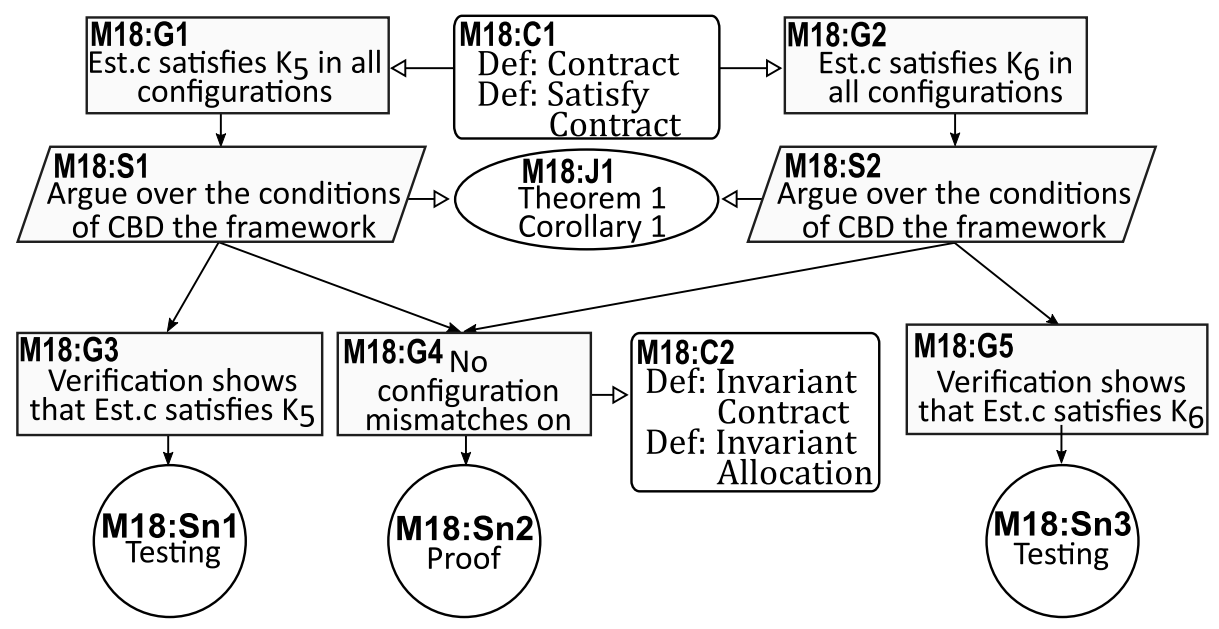

Figure 5.16: Content of module M18.

$\mathfrak{M}$, i.e. each presence condition defines a subset of the configurations defined by a variability model. Because each presence condition is effectively a partial configuration, the same techniques for verifying if a configuration is valid, can be used to verify if a presence condition is consistent with a variability model. Finally, Theorem 2 and Theorem 3, define the conditions under which it is guaranteed that each variant derived from a proper PL specification structure is a proper specification structure, which corresponds to a valid variant. The above argumentation can be expressed in the form of a GSN goal-structure to argue that product-line management mechanisms are effective and reliable.

The presented assurance case patterns yield the argumentation that the overall system, e.g. the FLD system, satisfies the allocated contract and that each constituent component, composed with other components of the system, satisfies the guarantees of the allocated contracts. This means that there is no module that contains the instantiation of the pattern in Figure 5.12 for the overall system, e.g. the FLD component. As mentioned, the reason for this is that the assumptions of the system, e.g. assumption $A_{1}$ of the FLD system, is effectively an assumption on the intended deployment environment, e.g. an assumption on the vehicle-driver. Although the pattern does not include the argumentation about the suitability of intended operating environment, because the assumptions of the system are explicitly declared, such argumentation could 


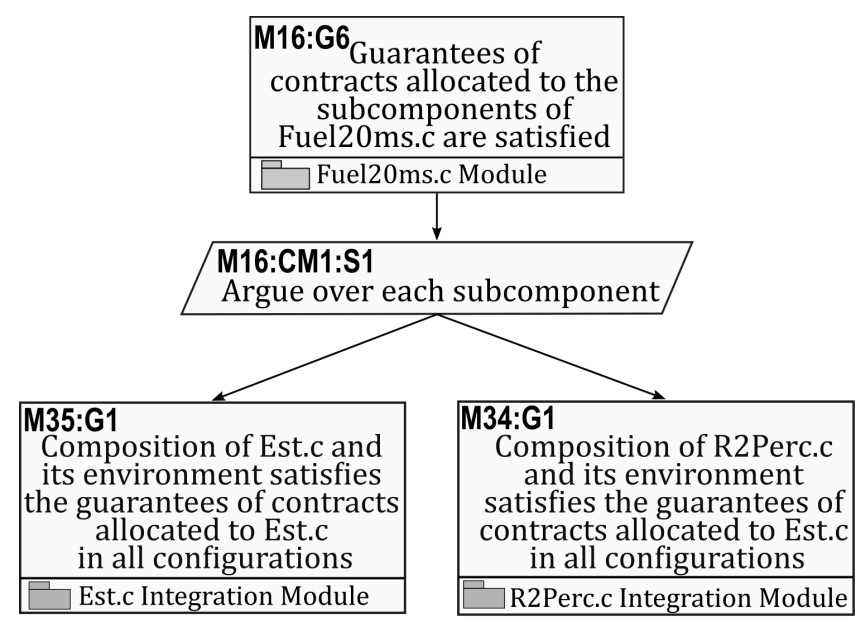

Figure 5.17: Content of GSN contract-module M16:CM1.

be developed, and connected to the argumentation based on the patterns in Figure 5.10 and Figure 5.10 . This also means that if during deployment it is realized that the real-world environment exhibits new or different behavior than expected, the system assumptions will undergo changes and this will also have a traceable impact on the assurance case.

\section{Discussion}

In this section we answer research question RQ1 and RQ2 by considering the results of the exploratory case study, and by analyzing the assurance-case pattern from the point of view of the claimed benefits of the modular architecture, and from the point of view of feasibility to produce the required evidence.

\subsection{Answering RQ1}

The first research question asked if the CBD framework can be used to model arbitrary product lines and analyze if all variants satisfy a particular property without performing the analysis per-variant. Given the abstract nature of the CBD framework, the product-line extension of the CBD framework, and the results of applying the $\mathrm{CBD}$ framework to a real, industrial configurable systems, 
there is a strong indication that the answer to RQ1 is positive, but a definitive answer would require further validation. Also, there are several notable facets to the answer to RQ1.

From the expressiveness perspective, we argue that the CBD framework is capable of representing an arbitrary product line. As was shown in the case study, the analyzed artifacts contained entities that could be directly interpreted as components, specifications, and presence conditions, and in general these concepts are widely used independently of the specific notation. Moreover, although not developed according to the CBD framework, the analyzed requirements documents already contained some contract-like requirements, which means that contract-like thinking is not uncommon. For example, components bought from suppliers were specified in terms of contracts. Traceability links that corresponds to composition and allocation relations were also explicitly declared in the analyzed documents. Finally, the refines relations were not explicitly present in the analyzed documents, but on a positive note, an attempt to make them explicit revealed a mismatch between the high-level design and system implementation.

On the other hand, in this particular case, the effort to use the CBD framework was not negligible. However, as discussed in Section 6.4 the reason for this were primarily the engineering practices and the quality of the available artifacts and traceability links for the analyzed system. Conceptually, the conditions of the CBD framework enforce rigorous development with detailed architecture, specifications, and traceability links. If this is not deemed as needed, then using the CBD framework might require too much effort compared to the possible benefits. However, for safety-critical system, rigorous development process is a requirement, thus CBD-framework conditions provide valuable guidance.

This can be seen by inspecting the requirements of the functional-safety standard for the automotive domain, ISO 26262. Namely, as already discussed in Section 4 the concept of a specification structure already matches well with the idea of ISO 26262 where higher level requirements are broken down into lower level requirements, in parallel with the development of system components. When it comes to the management of configurable systems, ISO 26262 recognizes that an item can exist in multiple variants, and that the possible variants should be considered during system design, implementation, and verification. If considering all variants is not feasible, then considering a reasonable, justified, and representative subsets may be sufficient, where such subsets are primarily selected using engineering judgment. However, the standard does not offer guidance about what is reasonable, justified, or representative, while engineering judgment is necessarily subjective. More detailed guidance exists for software where configuration data, which specifies the possible variants, must 
be consistent with the declared requirements, and it must be itself consistent. Furthermore, if there are calibration variables that impact the software runtime behavior, these must be consistent with the configuration data, declared requirements etc. However, the concept of consistency is not precisely defined.

The conditions of the extended CBD framework offer a technical solution for the above general concepts recognized by ISO 26262. Firstly, because the framework allows arguing about all variants, the need to identify a reasonable or a representative subset is avoided. Secondly, the concept of presence conditions can be used to capture the variability of all components, and not only software components. Thirdly, the invariance conditions from Def. 14.Def. 16 and from Theorem 3 directly correspond to the concept of consistency between the various types of artifacts that apply to multiple variants.

\subsection{Answering RQ2}

The second research question asked how a CBD model of a product line can be used to construct the corresponding assurance case. To answer RQ2, Section 7 presented two modular, assurance-case patterns, which define how a CBD model of product line can be used to construct the corresponding assurance case argumentation. However, because the pattern could have been defined differently to express the same argument, the following subsections discuss whether the modular architecture truly facilitates the stepwise creation and maintenance of an assurance case, and whether the required evidence is feasible to produce in a realistic engineering context.

\section{Benefits of the Modular Architecture}

To facilitate the management of assurance cases of realistic size, the pattern from Figure 5.10 and Figure 5.10 enforces a modular architecture. Firstly, having a coarse-grained representation of an assurance case in terms of modules, facilitates assurance-case maintenance and review on a high level. Secondly, as shown in Figure 5.13 , the modules are created for components at different abstraction levels, as defined for a particular system. In this sense, each module and the contained argumentation correspond to assurance claims and evidence originating from a particular development phase, which are possibly concurrent. At the same time, the usage of GSN contract modules allows the creation of modules independently, and then the modules can be related once the contract-modules are instantiated. Also note that because the modules capture the argumentation for particular components, the modules can be used to assign the responsibilities 
for their creation and maintenance. For example, module M18 in Figure 5.13 would probably be under the responsibility of a small development group, while modules M16 and M35 would be under the responsibility of the group responsible for the COO SW. Finally, the separation into two patterns, contained by two separate modules, directly supports the idea of safety element out of context (SEooC), as defined by ISO 26262. The purpose of SEooC is to allow suppliers to develop safety-critical components with respect to a contract provided by their customers, while the supplier's customers are responsible for the correct integration of SEooC components into their systems. In summary, despite the need to further validate the approach on full-size systems, the current modular architecture suggests that the creation of the argumentation within the modules can be tightly integrated with a typical development process.

Although widely considered beneficial, an industrial evaluation [55] reports two major challenges when using modular argumentation: (i) deciding on the content of modules such that references between the modules, the so-called module interface, are clearly defined, and (ii) ensuring that the composition of all modules yields a valid argument because the assumptions, context, and evidence between different modules might be incompatible. The present paper overcomes these two challenges by relying on the CBD framework. Firstly, each supportedBy relation between two modules, as defined by corresponding contract-modules, corresponds to the composition of components with respect to the guarantees relevant for a particular instantiation of pattern in Figure 5.12 Regarding the second challenge, the modularization in Figure 5.13 is simply a distribution of arguments about premises of Corollary 1 across multiple modules, but the overall argument is preserved and thus also the argument validity.

\section{Feasibility of Producing the Required Evidence}

This section considers each type of evidence required by the assurance-case pattern from Figure 5.10 and Figure 5.10 and discusses the state-of-the-art techniques to produce such evidence, and the state-of-practice in SCANIA. In other words, given that the each evidence corresponds to an analysis of the constructed PL specification structure with respect to the conditions of Corollary 1 . this section discusses how to practically analyze a PL specification structure.

Absence of configuration mismatches. Evidence such as M16:Sn1, or M35:Sn1 or M18:Sn2, is produced by analyzing the constructed PL specification structure against conditions i) and ii) of Corollary 1. The work in 64 shows how such evidence can be produced using description logic. In general, 
such evidence can be routinely performed by using basic SMT solving [17]. For example, in the context of the model in Figure 5.8, verifying if the allocation of $\left(A_{5}, G_{4}\right)$ to component $C_{\text {Fuel2oms }}$ is invariant (c.f. Def. 15), means verifying if $p\left(\mathrm{G}_{4}\right)=_{\Theta} p\left(\mathrm{C}_{\text {Fue120ms }}\right)$, i.e. $\varphi_{27} \models_{\Theta} \varphi_{7}$. Practically, this implies building an SMT model that contains each $\theta_{i} \in \Theta$ from a variability model, the assertion $\neg\left(\varphi_{27} \rightarrow \varphi_{7}\right)$, and checking if such SMT model is satisfiable. If the model is satisfiable, then the allocation is not invariant, and vice versa. Note that although the $\Theta$ of a large variability model contains many constraints and consequently results in a large SMT model, once each $\theta_{i} \in \Theta$ is asserted, verifying different invariance constraints requires changing a single expression, namely the negated implication between presence conditions, i.e. $\neg\left(\varphi_{j} \rightarrow \varphi_{k}\right)$. Also, as shown in [59, this type of satisfiability checks is tractable in practice. In summary, there are well-known, scalable techniques to produce this type of evidence, but as discussed in Section 6.4. SCANIA has not adopted these.

Conformance with architectural principles. Producing the evidence such as M16:Sn2 in Figure 5.14, which corresponds to the analysis of the constructed PL specification structure against the conditions of Def. 12, can be more or less challenging. Because the design and implementation happen in multiple phases that are supported by different tools, and performed by different engineering groups, ensuring that the same principles are uniformly followed is challenging. If it is possible to extract, merge, and possibly transform the relevant artifacts into a formal model, then this evidence can be produced automatically. Under such assumptions, the work in 64] presents a method based on description logic to produce such evidence in an automated way. Additional tools that could probably be used to perform such analysis are Alloy [49] or Clafer [5].

Previous approaches for single system assurance [23, 43, 39] frame this type of problem as the central activity in Model-Based Engineering (MBE) paradigm, namely the instantiation of a meta-model into a model. While such approaches also promise high levels of automation, they may not be always applicable because the engineering context might not be fully MBE compliant. In the FLD example, the logical architecture of the FLD system was represented by a welldefined model (c.f. Table 5.1), and the corresponding tool performed consistency checks, but there was no model of the software architecture.

Specification refinement. Regarding the evidence about specification refinement, e.g. solution elements M16:Sn3 and M35:Sn2 in Figure 5.15, this corresponds to the analysis of the constructed PL specification structure against 
condition iii) of Corollary 1 . In general, this is a hard problem for any method. Usually, it is more likely that implementation-level specifications, e.g. the ones allocated to software or hardware components, are susceptible to the application of formal methods that can prove refinement because they are typically more detailed. The work in [27] introduces an automated, SMT-based method to verify specification refinement, and exemplifies the approach also on the FLD system. However, it is a widely acknowledged fact that writing formal requirements is a difficult and tedious task, thus in a realistic industrial context, expert review will probably be the verification method of choice. Note that among the analyzed FLD documents, there was no explicit evidence of specification refinement, but each requirement document had to be approved by a senior engineer. Thus, this type of evidence is probably implicitly created, although without documenting the reasons to approve or reject a requirement document.

Component behavior. The fourth and final type of evidence, e.g. solution elements M18:Sn1 and M18:Sn3 in Figure 5.16, is about showing that each contract allocated to an atomic component is satisfied, and this corresponds to analyzing the PL specification structure against condition iv) of Corollary 1. As with other analyses, depending on the level of formality, this analysis can be more or less automated. In the case of software components where the assumptions and guarantees are expressed in a formal language, formal softwareverification approaches against contract-based specifications can often produce good results [37. On the other hand, the most common, and a de-facto standard method in industry, to verify component behavior is unit and integration testing. The analyzed FLD documentation confirms this as it contained reports of extensive testing. Note that because argumentation based on the CBD framework requires only the verification of atomic component, strictly speaking, this means that there is no need for integration testing. However, because the complexity of systems can lead to unforeseen interactions between components, integration testing can be used as a method to ensure absence of unwanted interactions [58]. For example, goal M35:G1 in Figure 5.15 could be supported by an additional argumentation leg, that claims that no unwanted interactions were observed, supported by integration-testing results.

\section{Related Work}

Section 1.1 has already discussed two approaches that are directly related to the presented approach. However, there are other approaches that tackle the 
challenge of representing a product line, or the challenge of argumentation reuse, potentially in assurance cases for product lines.

\subsection{Frameworks to Represent a Product Line}

One line of research uses the ideas of model-based engineering to represent a product line, typically as a series of UML [86, 33, 52] or SysML [10, 29] models by defining stereotypes to support product line engineering. Both UML and SysML are comprehensive, semi-formal modeling frameworks that support a wide range of concrete artifacts and traceability links, definitively a greater number than the CBD framework. However, unlike the CBD framework, UML and SysML do not support compositional reasoning, and this the fundamental reason why any analysis must be performed per-variant. Furthermore, although UML and SysML are mature frameworks for everyday engineering, assuming a representation of a product line in a concrete engineering framework such as UML or SysML would implicitly place requirements on the development methods and process, and a major part of the motivation to use the CBD framework is exactly to avoid doing so.

Another line of research comes from the domain computer science [12, 14, 4], where various types of labeled transition systems are used to model system variants in terms of their states, and transitions between the states, where the transitions are labeled with presence conditions. Then, formal specifications, expressed in various types of logic, are verified over the transition systems by using model checking techniques. Due to their abstract and formal nature, such frameworks can be used to represent and rigorously analyze arbitrary systems. Moreover, compositional reasoning is well-defined for transition systems, i.e. it is possible to compose transition systems and deduce the properties of the resulting composition. However, on a practical level, such approaches abstract away the traceability links between the artifacts such as allocated to, or intended refinement from the CBD framework. Moreover, because of their fully formal nature, it is unlikely that a significant number of components will be represented as transitions systems and that a significant number of specifications will be expressed as logical formulas instead of the typically informal and semi-formal formats 84. Finally, as is often the case, this level of formality is achieved by trading expressiveness, i.e. concrete logical languages allow expressing a restricted class of specifications, and transition systems allow the modeling only of discrete systems, while the CBD framework makes no such assumptions. 


\subsection{Argumentation Reuse}

Two schools of thought exist for the construction of argumentation structures. The first line of research has focused on capturing assurance case patterns [20] that have been used in real world assurance cases or in case studies performed by researchers. The second line of research has focused on the creation of assurancecase arguments by analyzing various types of artifacts. In the context of product lines, besides the approaches described in the introduction [38, 41, 18, 45, 46, 47], most notable contributions come from the latter group [31, 74, 73, 16, 32, 50].

The work in 31 presents a method called VROOM and $c C$, which focuses on achieving compliance of a product line with the first two phases of the ISO 26262 standard in the automotive domain. The main idea of the approach is to align each step of the safety lifecycle proposed by ISO 26262, with the productline engineering idea of modeling common and variable parts of engineering assets within a product line. The idea leads to a double, $V$ development model, where in each phase of the model, there is a set of recommended, tool-supported, engineering activities which should enable systematic, fine-grained, tracing of engineering assets within a product line. Compliance with the first two phases of ISO 26262 is shown by leveraging the established traceability to create an assurance case which reflects the variability within the engineering assets. In other words, this approach follows the same idea as the work in [38, 41, 18], where the assurance case arguments are for all system variants are syntactically integrated, but it is assumed that per-variant arguments are derived and analyzed if they are valid and convincing.

The line of work in [74, 73] provides an approach for the creation of reusable assurance cases fragments based on a semi-formal notion of weak and strong safety contracts. The approach considers the concept of Safety Element out of Context (SEooC) from the standard ISO 26262, which is used to represent the components that OEMs procure from external suppliers. The essence of the approach is to perform hazard analysis and risk assessment for a SEooC using a variant of Fault Propagation and Transformation Calculus (FPTC) [30] and then transform the analysis results into safety contracts. The present paper considers development and assurance of arbitrary product lines, and assurance of arbitrary dependability properties, from the OEM perspective, and because of that relies on a more expressive contract-based framework which subsumes the weak and strong safety contracts. Consequently, the approach in the present paper argues about system-level dependability properties instead of only safety of SEooC. Furthermore, unlike the approach in [73] where presence conditions are assumptions of contracts, the presence conditions in the present paper are 
orthogonal to components and specifications, thus allowing presence conditions of artifacts and the artifact themselves to evolve separately.

Unlike the work in the present paper, which focuses on product-based arguments, the line of work in [32, 50] focuses explicitly on process-based arguments that can be reused. The reuse can occur between different yet similar systems, and in that sense this work is applicable to product-line engineering. However, reuse of process arguments can also occur between different domains, regulated by different standards [32]. The central artifact in this line of work is a model of the used engineering processes, with variable process steps, expressed in the SPEM format [67]. The variability of the process is modeled either with the build-in capabilities of SPEM format [32], or using the integration between the BVR language 42, and the implementation of SPEM within the EPF composer ${ }^{1}$ Once the process model is in place, the so-called process line, variants of the process can be derived, and process-based arguments can be constructed for different process variants. Moreover, the approach can be also used to detect some types of fallacies within process-based arguments [63.

Finally, it should be noted that the work from [32, [50, 74] has been integrated into a tool ecosystem AMASS [16], which support additional methods for analysis and assurance of arbitrary dependability properties of single systems. For example, the work in [51] exploits the AMASS tool ecosystem to show how impact analysis can be performed between a product-line and a process-line specification in the BVR and the EPF tool.

\section{Threats to Validity}

Construct Validity. The answers to research question depend on the results of the exploratory case study. To avoid the risk of analyzing the wrong artifacts, e.g. mismatching versions, domain experts were included in all phases of the study before starting the CBD model synthesis. Because the CBD concepts such as component, specification, allocated to relation etc., are generic, and defined so that they match closely to engineering practices, the risk of misinterpreting an artifact as a CBD concept was low. For example, the C-language implementation contains $\mathrm{C}$-functions, which were mapped to components $\mathrm{C}_{\mathrm{R} 2 \mathrm{Perc}}$ and $\mathrm{C}_{\mathrm{Est}}$, $\mathrm{a}^{*}$.c file of the software-application component is mapped to $\mathrm{C}_{\mathrm{Fuel} 20 \mathrm{~ms}}$, the analyzed version of the COO source code is mapped to $\mathrm{C}_{\mathrm{CoOSW}}$ etc. Similarly, each considered requirement from requirements documents was directly mapped to a single specification $S$.

\footnotetext{
1 https://www.eclipse.org/epf/
} 
Internal Validity. To minimize the risk of using an unsuitable framework for product-line modeling and analysis, Section 9.1 compares the CBD framework to other feasible frameworks. When the initial mapping of the analyzed artifacts did not yield a proper PL specification structure, it was decided to infer the missing information instead of using a method to elicit the information from the domain experts. In this way, we avoided any bias that domain experts could have introduced, and also avoided the risk that the domain experts misinterpret the conditions of the CBD framework. Given that the authors defined the inference rules, and to avoid any bias during the inference of the missing information, we took care that no new knowledge is introduced. Because of that, as can be seen in Table 5.2, each inferred specification is always equivalent to an explicitly declared SCANIA requirement. Moreover, the inference process was guided by explicitly, and formally defined conditions of the CBD framework, which further minimizes the risk of introducing bias. It should be noted that during the inference process, a slightly different set of inferences was possible. For example, Table 5.2 states that $\mathrm{G}_{1} \equiv \mathrm{G}_{2} \equiv \mathrm{G}_{3}$, where $\mathrm{G}_{2}$ and $\mathrm{G}_{3}$ were inferred to satisfy Def. 9 [ii) and Corollary 1 iii) although the edge $e^{\mathrm{r}}=\left(\mathrm{G}_{4}, \mathrm{G}_{3}\right)$ violates Corollary 1 iii), Alternatively, it could have been inferred that $\mathrm{G}_{2} \equiv \mathrm{G}_{3} \equiv \mathrm{G}_{4}$, which also leads to a proper PL specification structure, but then edge $e^{\mathrm{r}}=\left(\mathrm{G}_{2}, \mathrm{G}_{1}\right)$ would violate Corollary 1 iii). In other words, because no new knowledge is added, and the same inference rules are used, different alternatives would still lead to a proper PL specification structure, and to the violation of the same semantic conditions, e.g. the ones in Corollary 1.

External Validity. The case-study system, namely the FLD system, is a typical automotive system. It is a mature, currently in-production system with thousands of unique system-variants. The system includes various types of components, e.g. software, hardware, and mechanical, and it is also safety-critical which suggests that an assurance case should be developed for it. Moreover, the FLD system proved to be a particularly challenging system for an application of the CBD framework because the used engineering practices were not aligned with the practices recommended by the relevant safety standard, which are supported by the CBD framework. However, it was still possible to apply the CBD framework. These factors strongly suggest that the extended CBD framework is able to model and support the analysis of arbitrary product lines.

Reliability. To enable the reproducibility of the exploratory study, and the applicability of the inference steps, the applied methodology was described in 
detail in Section 6.1. Moreover, for each entity and traceability link that was inferred, there is an explicit description about how that was achieved. One limitation to the reliability of the study is the inability share the raw SCANIA artifacts due to confidentiality issues. This limitation was partly alleviated by providing an online repository with assurance-case related artifact such as the assurance-case pattern, the complete modular architecture for the FLD system.

\section{Conclusion}

To satisfy the needs of as many customers as possible, enterprises are increasing the allowed levels of customization, even for complex cyber-physical systems that must satisfy critical dependability properties such as safety, security or reliability. Making such systems highly configurable, e.g. engineering them as product lines, hinders the use of existing, single-system assurance methods because analyzing and assuring individual system configurations becomes infeasible.

This paper has introduced a novel and general approach for the creation of an assurance case for a complete product line, instead of for each individual system configurations. The first contribution of the paper is a product-line extension of a general-purpose contract-based design-framework, in order to obtain a rigorous, holistic model of all system configurations within a product line. The result of this extension is Corollary 1, which summarizes the conditions under which it can be deduced that all system configurations satisfy a particular critical property. The applicability of the extended framework is evaluated through an exploratory case study on a part of a real product-line from ScAnia. The second contribution defines a modular, assurance-case pattern, which is based on the conditions from Corollary 1, and which can be instantiated to create product-based argumentation about a complete product line. A comparison to the ISO 26262 guidelines, as well with observed practices in SCANIA shows that the usage of the pattern can be aligned with a typical engineering process. Also, the majority of the required evidence is either already typically produced, or it can be produced with well-known automated techniques.

\section{Acknowledgment}

D. Nešić and M. Nyberg were supported by the ECSEL PRYSTINE project (No 783190) and by SCANIA CV AB. B. Gallina was partly supported by ECSEL AMASS project (No 692474). 


\section{Bibliography}

[1] Online appendix.

[2] Adelard LLP. ASCAD: Adelard Safety Case Development Manual. Technical report, 1998.

[3] Sven Apel, Don Batory, Christian Kästner, and Gunter Saake. Featureoriented software product lines. Springer, 2016.

[4] P. Asirelli, M. H. ter Beek, S. Gnesi, and A. Fantechi. Formal description of variability in product families. In International Software Product Line Conference (SPLC), pages 130-139, 2011.

[5] Kacper Bąk, Zinovy Diskin, Michał Antkiewicz, Krzysztof Czarnecki, and Andrzej Wąsowski. Clafer: unifying class and feature modeling. Software 83 Systems Modeling, 15(3):811-845, 2016.

[6] David Benavides, Sergio Segura, and Antonio Ruiz-Cortés. Automated analysis of feature models 20 years later: A literature review. Information Systems, 35(6):615-636, September 2010.

[7] Saddek Bensalem, Marius Bozga, Joseph Sifakis, and Thanh-Hung Nguyen. Compositional verification for component-based systems and application. In International Symposium on Automated Technology for Verification and Analysis, pages 64-79. Springer, 2008.

[8] Albert Benveniste, Benoît Caillaud, Dejan Nickovic, and et al. Contracts for system design. Foundations and Trends in Electronic Design Automation, 12:124-400, 2018. 
[9] Roderick Chapman and Florian Schanda. Are we there yet? 20 years of industrial theorem proving with spark. In Interactive Theorem Proving (ITP), pages 17-26. Springer, 2014.

[10] A. G. Chiquitto, I. M. S. Gimenes, and E. Oliveira. SyMPLES-CVL: A SysML and CVL Based Approach for Product-Line Development of Embedded Systems. In Brazilian Symposium on Components, Architectures and Reuse Software, pages 21-30, 2015.

[11] A. Cimatti and S. Tonetta. A property-based proof system for contractbased design. In Euromicro Conference on Software Engineering and Advanced Applications (SEAA), pages 21-28. IEEE, 2012.

[12] Andreas Classen, Maxime Cordy, Pierre-Yves Schobbens, Patrick Heymans, Axel Legay, and Jean-Francois Raskin. Featured transition systems: Foundations for verifying variability-intensive systems and their application to ltl model checking. IEEE Transactions on Software Engineering, 39(8):10691089, 2012.

[13] Paul Clements and Linda Northrop. Software Product Lines: Practices and Patterns. Addison-Wesley Professional, 2001.

[14] Maxime Cordy, Pierre-Yves Schobbens, Patrick Heymans, and Axel Legay. Beyond boolean product-line model checking: dealing with feature attributes and multi-features. In International Conference on Software Engineering (ICSE), pages 472-481. IEEE, 2013.

[15] Krzysztof Czarnecki, Paul Grünbacher, Rick Rabiser, Klaus Schmid, and Andrzej Wassowski. Cool features and tough decisions: a comparison of variability modeling approaches. In International workshop on variability modeling of software-intensive systems (VAMOS), pages 173-182. ACM, 2012.

[16] Jose Luis de la Vara, Alejandra Ruiz, and Barbara et al. Gallina. The AMASS Approach for Assurance and Certification of Critical Systems. In Embedded World 2019, 2019.

[17] Leonardo De Moura and Nikolaj Bjørner. Z3: An efficient smt solver. In International conference on Tools and Algorithms for the Construction and Analysis of Systems (TACAS), pages 337-340. Springer, 2008. 
[18] André L. de Oliveira, Rosana T. V. Braga, Paulo C. Masiero, Yiannis Papadopoulos, Ibrahim Habli, and Tim Kelly. Supporting the automated generation of modular product line safety cases. In International Conference on Dependability and Complex Systems (DePCoS-RELCOMEX), pages 319-330. Springer, 2015.

[19] Willem-Paul De Roever, Hans Langmaack, and Amir Pnueli. Compositionality: The Significant Difference: International Symposium, COMPOS. Springer, 2003.

[20] Ewen Denney and Ganesh Pai. A formal basis for safety case patterns. In Computer Safety, Reliability, and Security (SAFECOMP), pages 21-32. Springer, 2013.

[21] Ewen Denney and Ganesh Pai. Tool support for assurance case development. Automated Software Engineering, 25(3):435-499, 2018.

[22] Christian Dietrich, Reinhard Tartler, Wolfgang Schröder-Preikshat, and Daniel Lohmann. Understanding linux feature distribution. In Workshop on Modularity in Systems Software (MODULARITY), pages 15-20. ACM, 2012 .

[23] Zinovy Diskin, Tom Maibaum, Alan Wassyng, Stephen Wynn-Williams, and Mark Lawford. Assurance via model transformations and their hierarchical refinement. In International Conference on Model Driven Engineering Languages and Systems (MODELS), pages 426-436. ACM, 2018.

[24] Yael Dubinsky, Julia Rubin, Thorsten Berger, Slawomir Duszynski, Martin Becker, and Krzysztof Czarnecki. An exploratory study of cloning in industrial software product lines. In European Conference on Software Maintenance and Reengineering (CSMR), pages 25-34. IEEE, 2013.

[25] Sascha El-Sharkawy, Adam Krafczyk, and Klaus Schmid. An empirical study of configuration mismatches in linux. In International Systems and Software Product Line Conference (SPLC), pages 19-28. ACM, 2017.

[26] Railway applications - communication, signaling and processing systems safety related electronic systems for signaling. standard, European Committee for Electrotechnical Standardization, 2003.

[27] Predrag Filipovikj, Guillermo Rodriguez-Navas, Mattias Nyberg, and Cristina Seceleanu. Automated smt-based consistency checking of industrial critical requirements. 17(4):15âĂŞ28, 2018. 
[28] Thomas Fogdal, Helene Scherrebeck, Juha Kuusela, Martin Becker, and Bo Zhang. Ten years of product line engineering at danfoss: lessons learned and way ahead. In International Systems and Software Product Line Conference (SPLC), pages 252-261. ACM, 2016.

[29] Jesús Padilla Gaeta and Krzysztof Czarnecki. Modeling aerospace systems product lines in sysml. In International Conference on Software Product Lines (SPLC), pages 293-302. ACM, 2015.

[30] B. Gallina, M. A. Javed, F. U. Muram, and S. Punnekkat. A model-driven dependability analysis method for component-based architectures. In Euromicro Conference on Software Engineering and Advanced Applications (SEAA), pages 233-240. IEEE, 2012.

[31] Barbara Gallina, Antonio Gallucci, Kristina Lundqvist, and Mattias Nyberg. VROOM \& cC: a Method to Build Safety Cases for ISO 26262compliant Product Lines. In Conference on Computer Safety, Reliability and Security (SAFECOMP): SASSUR Workshop. HAL, 2013.

[32] Barbara Gallina, Shaghayegh Kashiyarandi, Karlheinz Zugsbratl, and Arjan Geven. Enabling cross-domain reuse of tool qualification certification artefacts. In International Conference on Computer Safety, Reliability and Security (SAFECOMP), pages 255-266. Springer, 2014.

[33] Hassan Gomaa. Software Modeling and Design: UML, Use Cases, Patterns, and Software Architectures. Cambridge University Press, 2011.

[34] Janusz Górski, Aleksander Jarzębowicz, Jakub Miler, and et al. Supporting assurance by evidence-based argument services. In International Conference on Computer Safety, Reliability, and Security (SAFECOMP), pages 417-426. Springer, 2012.

[35] Susanne Graf and Sophie Quinton. Contracts for bip: Hierarchical interaction models for compositional verification. In Forum for Fundamental Research on Theory, Models, and Application for distributed systems, pages 1-18. Springer, 2007.

[36] Object Management Group. Structured Assurance Case Metamodel. standard, Mar 2019. Beta version.

[37] Dilian Gurov, Christian Lidström, Mattias Nyberg, and Jonas Westman. Deductive functional verification of safety-critical embedded c-code: An 
experience report. In Critical Systems: Formal Methods and Automated Verification Workshop, pages 3-18. Springer, 2017.

[38] Ibrahim Habli. Model-based assurance of safety-critical product lines. $\mathrm{PhD}$ thesis, Department of Computer Science, University of York, York, UK, 2009 .

[39] Ibrahim Habli, Ireri Ibarra, Roger S Rivett, and Tim Kelly. Model-based assurance for justifying automotive functional safety. Technical report, SAE Technical Paper, 2010.

[40] Ibrahim Habli and Tim Kelly. Process and product certification arguments: Getting the balance right. SIGBED Review, 3(4), 2006.

[41] Ibrahim Habli and Tim Kelly. A safety case approach to assuring configurable architectures of safety-critical product lines. In International Symposium on Architecting Critical Systems (ISARC 10), pages 142-160. Springer, 2010.

[42] Øystein Haugen and Ommund Øgård. BVR - Better Variability Results. In System Analysis and Modeling: Models and Reusability, pages 1-15. Springer, 2014.

[43] Richard Hawkins, Ibrahim Habli, Dimitris Kolovos, Richard Paige, and Tim Kelly. Weaving an assurance case from design: a model-based approach. In International Symposium on High Assurance Systems Engineering (HASE), pages 110-117. IEEE, 2015.

[44] Arnaud Hubaux, Yingfei Xiong, and Krzysztof Czarnecki. A user survey of configuration challenges in linux and ecos. In International Workshop on Variability Modeling of Software-Intensive Systems (VAMOS), pages 149155. ACM, 2012.

[45] Stuart Hutchesson and John McDermid. Development of high-integrity software product lines using model transformation. In Computer Safety, Reliability, and Security (SAFECOMP), pages 389-401. Springer, 2010.

[46] Stuart Hutchesson and John McDermid. Towards cost-effective highassurance software product lines: The need for property-preserving transformations. In International Software Product Line Conference (SPLC), pages 55-64. IEEE, 2011. 
[47] Stuart Hutchesson and John McDermid. Trusted product lines. Information and Software Technology, 55:525 - 540, 2013.

[48] International Organization for Standardization. ISO 26262: Road vehicles - Functional safety, Nov 2011.

[49] Daniel Jackson. Alloy: a lightweight object modelling notation. ACM Transactions on Software Engineering and Methodology (TOSEM), 11(2):256-290, 2002.

[50] Muhammad Atif Javed and Barbara Gallina. Safety-oriented process line engineering via seamless integration between epf composer and bvr tool. In International Systems and Software Product Line Conference (SPLC) Volume 2, pages 23-28. ACM, 2018.

[51] Muhammad Atif Javed, Barbara Gallina, and Anna Carlsson. Towards variant management and change impact analysis in safety-oriented processproduct lines. In Symposium on Applied Computing (SAC), pages 23722375. ACM, 2019.

[52] Edson A. Oliveira Junior, Itana M. S. Gimenes, and JosÃl' C. Maldonado. Systematic Management of Variability in UML-based Software Product Lines. Journal of Universal Computer Science, 16(17):2374-2393, 2010.

[53] Christian Kästner and Sven Apel. Integrating compositional and annotative approaches for product line engineering. In Workshop on Modularization, Composition and Generative Techniques for Product Line Engineering, pages 35-40, 2008.

[54] T. P. Kelly and J. A. McDermid. Safety case construction and reuse using patterns. In Computer Safety, Reliability, and Security (SAFECOMP), pages 55-69. Springer, 1997.

[55] Tim Kelly and Simon Bates. The costs, benefits, and risks associated with pattern based and modular safety case development. In $U K M o D$ Equipment Safety Assurance Symposium (ESAS). UK Ministry of Defense, 2005.

[56] Jörg Liebig, Sven Apel, Christian Lengauer, Christian Kästner, and Michael Schulze. An analysis of the variability in forty preprocessorbased software product lines. In International Conference on Software Engineering-Volume (ICSE), pages 105-114. ACM, 2010. 
[57] Frank Linden, Klaus Schmid, and Eelco Rommes. Software Product Lines in Action: The Best Industrial Practice in Product Line Engineering. Springer, 2007.

[58] Leonardo Mariani, Sofia Papagiannakis, and Mauro Pezze. Compatibility and regression testing of cots-component-based software. In International Conference on Software Engineering (ICSE), pages 85-95. IEEE, 2007.

[59] Marcilio Mendonca, Andrzej Wąsowski, and Krzysztof Czarnecki. Satbased analysis of feature models is easy. In International Software Product Line Conference (SPLC), pages 231-240. ACM, 2009.

[60] Andreas Metzger and Klaus Pohl. Software product line engineering and variability management: achievements and challenges. In Proceedings of the Future of Software Engineering (FOSE), pages 70-84. ACM, 2014.

[61] Bertrand Meyer. Applying "design by contract". Computer, 25(10):40-51, October 1992.

[62] Mukelabai Mukelabai, Damir Nešić, Salome Maro, Thorsten Berger, and Jan-Philipp Steghöfer. Tackling combinatorial explosion: A study of industrial needs and practices for analyzing highly configurable systems. In International Conference on Automated Software Engineering ( $A S E)$, pages 155-166. ACM, 2018.

[63] Faiz UL Muram, Barbara Gallina, and Laura Gómez Rodríguez. Preventing omission of key evidence fallacy in process-based argumentations. In International Conference on the Quality of Information and Communications Technology (QUATIC), pages 65-73. IEEE, 2018.

[64] Damir Nešić and Mattias Nyberg. Verifying contract-based specifications of product lines using description logic. In International Workshop on Description Logic (DL), page 13, 2018.

[65] Damir Nešić, Mattias Nyberg, and Gallina Barbara. Constructing productline safety cases using contract-based specifications. In International Symposium on Applied Computing (SAC), pages 2022-2031. ACM, 2019.

[66] Damir Nešić, Jacob Krüger, Ştefan Stănciulescu, and Thorsten Berger. Principles of feature modeling. In Foundations of Software Engineering (FSE), pages 62-73. ACM, 2019. 
[67] Object Management Group. Software \& systems process engineering metamodel, 2008.

[68] Origin Consulting (York) Limited. GSN community standard version 2, Jan 2018.

[69] Thomas Rhodes, Frederick Boland, Elizabeth Fong, and Michael Kass. Software assurance using structured assurance case models. Journal of research of the National Institute of Standards and Technology, 115(3):209, 2010.

[70] Kenneth H Rosen and Kamala Krithivasan. Discrete mathematics and its applications: with combinatorics and graph theory. Tata McGraw-Hill Education, 2012.

[71] Marko Rosenmüller, Norbert Siegmund, Sven Apel, and Gunter Saake. Flexible feature binding in software product lines. Automated Software Engineering, 18:163-197, 2011.

[72] Per Runeson, Martin Host, Austen Rainer, and Bjorn Regnell. Case study research in software engineering: Guidelines and examples. John Wiley \& Sons, 2012.

[73] Irfan Sljivo, Barbara Gallina, Jan Carlson, and Hans Hansson. Configuration-aware contracts. In International Conference on Computer Safety, Reliability, and Security (SAFECOMP), pages 43-54. Springer, 2016.

[74] Irfan Sljivo, Barbara Gallina, Jan Carlson, Hans Hansson, and Stefano Puri. A method to generate reusable safety case argument-fragments from compositional safety analysis. Journal of Systems and Software, 131:570$590,2017$.

[75] Andreas Svendsen, Xiaorui Zhang, and et al. Lind-Tviberg. Developing a software product line for train control: A case study of cvl. In International Conference on Software Product Lines (SPLC), pages 106-120. Springer, 2010 .

[76] The International Electrotechnical Commission. ISO 61508: Functional Safety, 2010. 
[77] Thomas Thüm, Sven Apel, Christian Kästner, Ina Schaefer, and Gunter Saake. A classification and survey of analysis strategies for software product lines. ACM Computing Surveys (CSUR), 47(1):6, 2014.

[78] UL4600 Task Group. Ul4600 - standard for the evaluation of autonomous products. Technical report, April 2020.

[79] A. v. Rhein, A. Grebhahn, S. Apel, N. Siegmund, D. Beyer, and T. Berger. Presence-condition simplification in highly configurable systems. In International Conference on Software Engineering (ICSE), pages 178-188. ACM, 2015.

[80] Ran Wei, Tim P. Kelly, Xiaotian Dai, Shuai Zhao, and Richard Hawkins. Model based system assurance using the structured assurance case metamodel. Journal of Systems and Software, 154:211 - 233, 2019.

[81] Jonas Westman and Mattias Nyberg. Contracts for Specifying and Structuring Requirements on Cyber-Physical Systems, chapter 13. CRC Press, 2015 .

[82] Jonas Westman and Mattias Nyberg. Conditions of contracts for separating responsibilities in heterogeneous systems. Formal Methods in System Design, 52(2):147-192, Apr 2018.

[83] Jonas Westman and Mattias Nyberg. Preserving contract satisfiability under non-monotonic composition. In Formal Techniques for Distributed Objects, Components, and Systems (FORTE), pages 181-195. Springer, 2018.

[84] Jim Woodcock, Peter Gorm Larsen, Juan Bicarregui, and John Fitzgerald. Formal methods: Practice and experience. ACM Comput. Surv., 41(4), October 2009.

[85] Len Wozniak and Paul Clements. How automotive engineering is taking product line engineering to the extreme. In International Conference on Software Product Line (SPLC), pages 327-336. ACM, 2015.

[86] Tewfik Ziadi and Jean-Marc Jezequel. Software Product Line Engineering with the UML: Deriving Products, pages 557-588. Springer, 2006. 


\section{Theorem proofs}

This section presents the proofs of Theorem 1-3.

TheOREM 1. Let $\mathscr{A}$ be an architecture with the root $C_{r}$, and let $\mathcal{K}$ be a set of contracts such that each $K \in \mathcal{K}$ is allocated to at least one component from $\mathscr{A}$ and contract $K_{r} \in \mathcal{K}$ is allocated to $C_{r}$. Let $\mathcal{K}_{A t} \subseteq \mathcal{K}$ be the set of contracts allocated to atomic components $\mathcal{C}_{A t} \subseteq \mathscr{A}$. If

i) $\forall K \in \mathcal{K}_{A t} . \forall C \in \mathcal{C}_{A t} \cdot \operatorname{allTo}(K, C) \rightarrow C \vee K$,

ii) $\mathcal{K}$ forms a proper specification structure $\mathfrak{D}$,

iii) for each edge $e^{\mathrm{r}}=\left(S_{1}, S_{2}\right)$, it holds that $S_{1} \sqsubseteq S_{2}$,

then it holds that $C_{r}>K_{r}$.

Proof. To avoid notational overhead, we present the proof for a two-level architecture $\mathscr{A}$ with a root component $C_{r}$ whose subcomponents are atomic components. The proof for an architecture $\mathscr{A}$ with an arbitrary number of levels corresponds to applying the following reasoning to each composite component.

Let contract $K_{r}$ be such that it contains $n$ assumptions. According to Def. 7, we can rewrite the theorem claim as $\forall C_{e} . C_{e} \triangleright\left(A_{1}^{r} \sqcap \cdots A_{n}^{r}\right) \rightarrow C_{e} \otimes C_{r} \triangleright G_{r}$. Given that the theorem claim is an implication, we prove it by using a direct-argument strategy.

Assume an arbitrary $C_{e}$ such that $C_{e} \triangleright\left(A_{1}^{r} \sqcap \cdots A_{n}^{r}\right)$. From premise (ii) and according to Def. 9 ii), there exists a contract $\left(\mathcal{A}_{n}, G_{n}\right)$, allocated to a component $C_{m} \in \mathcal{C}_{A t}$, such the there exists an edge $e^{\mathrm{r}}=\left(G_{n}, G_{r}\right)$. Because $\mathcal{A}_{n}$ is possibly empty, there are two cases. In case a), $\mathcal{A}_{n}=\emptyset$ and from this and premise (i) it follows that $C_{m} \triangleright G_{n}$. From this and premise (iii), it follows that $C_{m} \triangleright G_{r}$. Because of monotonicity, $C_{m}$ can be composed with $C_{e}$ and components from $\mathcal{C}_{A t} \backslash C_{m}$ that comprise $C_{r}$, i.e. $C_{e} \otimes C_{r} \triangleright G_{r} \leftrightarrow C_{r} \triangleright K_{r}$. In case b), $\mathcal{A}_{n} \neq \emptyset$, and from premise (ii), according to Def. 9 i), it follows that for each $A_{j}^{n} \in \mathcal{A}_{n}$ there exists an edge $e^{\mathrm{r}}=\left(S, A_{j}^{n}\right)$ where $S \equiv A_{i}^{r}$, or $S \equiv G_{k}$ where $G_{k}$ is a guarantee of a contract $\left(\mathcal{A}_{k}, G_{k}\right)$ allocated to a component $C_{p}$, which is a sibling of $C_{m}$. If $S \equiv A_{i}^{r}$, given assumption $C_{e} \triangleright\left(A_{1}^{r} \sqcap \cdots A_{n}^{r}\right)$ and premise (iii), it follows that $C_{e} \triangleright A_{j}^{n}$ and from premise (i) it follows that $C_{e} \otimes C_{m} \triangleright G_{n} \leftrightarrow C_{e} \otimes C_{m} \triangleright G_{r}$. Similarly to case (i), due to monotonicity, $C_{e} \otimes C_{m}$ can be extended to $C_{e} \otimes C_{r} \triangleright G_{r}$. If $S \equiv G_{k}$, where $G_{k}$ is a guarantee of a contract $\left(\mathcal{A}_{k}, G_{k}\right)$, the previous reasoning process can be repeated for $\mathcal{A}_{k}$.

The previous steps can be repeated for each set of assumptions, such as $\mathcal{A}_{n}$, that are ancestors of $G_{r}$ in the graph of $\mathfrak{D}$. Because the graph of $\mathfrak{D}$ is finite and does not contain directed cycles, exploring all such assumptions must terminate and result in an expression $C_{x} \otimes \cdots \otimes C_{m} \triangleright G_{n}$. This expression either already contains $C_{e}$, or due to monotonicity it can be expanded with $C_{e}$ and any components that comprise $C_{r}$ but are not included in the expression. From this it follows that $C_{e} \otimes C_{r} \triangleright G_{n}$, and given premise (iii) if follows that $C_{e} \otimes C_{r} \triangleright G_{r} \leftrightarrow C_{r} \triangleright K_{r}$, which concludes the proof. 
Theorem 2 Let $\Gamma$ be a set of valid configurations, and let $\widehat{\mathfrak{D}}$ be a proper $P L$ specification structure. If

i) each contract is invariant,

ii) allocation of contracts to components from $\widehat{\mathscr{A}}$ is invariant,

iii) composition of child into parent components in $\widehat{\mathscr{A}}$ is invariant,

then each variant $\mathfrak{D}_{\gamma}$, is a specification structure.

Proof. Let $\gamma \in \Gamma$ be an arbitrary configuration, let $\mathfrak{D}_{\gamma}$ be the corresponding instantiated PL specification structure, and let $\varphi \in \Phi_{\widehat{D}}$ be a presence condition such that $\operatorname{eval}(\varphi, \gamma)=$ true. If $\varphi$ is the presence condition of some guarantee $G_{j}$, i.e. $p\left(G_{j}\right)=\varphi$, then from premise (i) (c.f. Def. 14 ii) it follows that each of the corresponding assumptions $A_{i}^{j} \in \mathcal{A}_{j}$ is such that $\operatorname{eval}\left(p\left(A_{i}^{j}\right), \gamma\right)=\operatorname{true}$ and the pair $\left(\mathcal{A}_{j}, G_{j}\right)$ is a contract. From this it follows that for each $A_{i}^{j} \in \mathcal{A}_{j}$ there exists a node $n_{i} \in \mathcal{N}_{\gamma}$ that represents $A_{i}^{j}$, and there exists a node $n_{j \neq i} \in \mathcal{N}_{\gamma}$ that represents $G_{j}$. Also, it follows that there exist edges $e^{\mathrm{a}}=\left(A_{i}^{j}, G_{j}\right)$ for each $A_{i}^{j} \in \mathcal{A}_{j}$. By using similar reasoning, and from premise (i) (c.f. Def. 14 i) , identical conclusions follow if $\varphi$ is the presence condition of some assumption $A_{i}^{j}$. Thus, given premises (i), and because according to Def. 11 the graph of $\widehat{\mathfrak{D}}$ conforms with the conditions from Def. 8 , it follows that the instantiated PL specification structure $\mathfrak{D}_{\gamma}$ forms a directed graph according to Def. 8

Def. 8 also requires that each contract from $\mathfrak{D}_{\gamma}$ is allocated to a at least one component from $\mathscr{A}_{\gamma}$, and that $\mathscr{A}_{\gamma}$ is an architecture according to Def. 4. Regarding contract allocation, from premise (ii), for each guarantee $G_{j}$ of a contract $\left(\mathcal{A}_{j}, G_{j}\right)$ such that $\operatorname{eval}\left(p\left(G_{j}\right), \gamma\right)=$ true, it follows that there exists a component $C_{c} \in \mathscr{A}_{\gamma}$ such that allTo $\left(\left(\mathcal{A}_{j}, G_{j}\right), C_{c}\right)$, and $\operatorname{eval}\left(p\left(C_{c}\right), \gamma\right)=$ true. From this, and regarding $\mathscr{A}_{\gamma}$ being an architecture according to Def. 4 , if $C_{c} \in \mathscr{A}_{\gamma}$ is the root of $\widehat{\mathscr{A}}$, and because $\mathscr{A}_{\gamma}$ is a tree (c.f. Def. 13iv) ), it follows that $\mathscr{A}_{\gamma}$ is an architecture according to Def. 4 for the configuration $\gamma$. If $C_{c}$ is a non-root component from $\widehat{\mathscr{A}}$, then from premise (iii) it follows that there exists a component $C_{p}$, which is the parent of $C_{c}$ in $\mathscr{A}_{\gamma}$, such that $\operatorname{eval}\left(p\left(C_{p}\right), \gamma\right)=$ true. Similarly, if $C_{p}$ is also a non-root component in $\widehat{\mathscr{A}}$, then from premise (iii) the presence condition of the parent of $C_{p}$ also evaluates to true. By repeating this process, it follows that the presence condition of component $C_{r}$, which is the root of $\mathscr{\mathscr { A }}$, evaluates to true and it holds that $C_{r} \in \mathscr{A}_{\gamma}$. From this, and because $\mathscr{A}_{\gamma}$ is a tree (c.f. Def. 13 iv) , it follows that $\mathscr{A}_{\gamma}$ is an architecture according to Def. 4 for configuration $\gamma$. This concludes the proof.

Theorem 3 Let $\Gamma$ be a set of valid configurations, and let $\widehat{\mathfrak{D}}$ be a proper $P L$ specification structure such that each variant $\mathfrak{D}_{\gamma}$ is a specification structure. If

i) for each $A_{i}^{j} \in \mathcal{S}$, and edges $e^{\mathrm{r}}=\left(S_{1}, A_{i}^{j}\right), \ldots,\left(S_{n}, A_{i}^{j}\right)$ it holds that $p\left(A_{i}^{j}\right) \models_{\Theta}$ $\bigvee_{k=1}^{n} p\left(S_{k}\right)$, 
ii) for each $G_{j} \in \mathcal{S}$, and edges $e^{\mathrm{r}}=\left(G_{1}, G_{j}\right), \ldots,\left(G_{n}, G_{j}\right)$ it holds that $p\left(G_{j}\right) \models_{\Theta}$ $\bigvee_{k=1}^{n} p\left(G_{k}\right)$,

then each $\mathfrak{D}_{\gamma}$ is proper specification structure.

Proof. The proof consists of showing that conditions from Def. 9 hold for each instantiation of $\widehat{\mathfrak{D}}$. Let $\gamma \in \Gamma$ be an arbitrary configuration and let $\mathfrak{D}_{\gamma}$ be an instantiation of $\widehat{\mathfrak{D}}$ for configuration $\gamma$. From premise (i) it follows that the instantiation $\mathfrak{D}_{\gamma}$ is such that for each $A_{i}^{j}$, where $\operatorname{eval}\left(p\left(A_{i}^{j}\right), \gamma\right)=\operatorname{true}$, there exists a specification $S_{k}$ such that $\operatorname{eval}\left(p\left(S_{k}\right), \gamma\right)=\operatorname{true}$. From this, and because $\mathcal{E}_{\gamma} \subseteq \mathcal{E}$ (c.f. Def. 13 ii) the edge $e^{\mathrm{r}}=\left(S_{k}, A_{i}^{j}\right)$ conforms to the condition (i) of Def. 9 . In other words, condition (i) from Def. 9 holds for $\mathfrak{D}_{\gamma}$.

The fact that condition (ii) from Def. 9 holds for $\mathfrak{D}_{\gamma}$ follows directly from premise (ii). Finally, according to premise (iii), $\mathfrak{D}_{\gamma}$ does not contain directed cycles. Consequently condition (iii) of Def. 9 holds. Given that $\gamma$ is arbitrary, the above proof holds for each instantiation of $\widehat{\mathfrak{D}}$. 